\title{
Recent theoretical and observational developments in magnetic helicity studies
}

\author{
P. Démoulin \\ Observatoire de Paris, section de Meudon, LESIA, UMR 8109 (CNRS), F-92195 \\ Meudon Cedex, France
}

\begin{abstract}
Magnetic helicity quantifies how the magnetic field is sheared and twisted compared to its lowest energy state (potential field). Such stressed magnetic fields are usually observed in association with flares, eruptive filaments, and coronal mass ejections (CMEs). Magnetic helicity plays a key role in magnetohydrodynamics because it is almost preserved on a timescale less than the global diffusion time scale. Its conservation defines a constraint to the magnetic field evolution.

Only relatively recently, scientists have realized that magnetic helicity can be computed from observations, and methods have been derived to bridge the gap between theory and observations. At the photospheric level, the rate (or flux) of magnetic helicity can be computed from the evolution of longitudinal magnetograms. The coronal helicity is estimated from magnetic extrapolation, while the helicity ejected in magnetic clouds (interplanetary counter-part of CMEs) is derived through modelling of in-situ magnetic field measurements. Using its conserved property, a quantitative link between phenomena observed in the corona and then in the interplanetary medium has been achieved.
\end{abstract}

Key words: magnetic fields, magnetic helicity, magneto-hydrodynamic, flares, coronal mass ejections, magnetic clouds

\section{Why analyze magnetic helicity ?}

Magnetic helicity quantifies how the magnetic flux tubes are wounded around each other, with the larger contribution coming from flux tubes having the larger magnetic flux. In simple examples it measures how much a flux tube is twisted, or how much a magnetic arcade is sheared, or how many times two closed flux tubes turned around each other. Unlike magnetic shear or twist, magnetic helicity can be precisely defined in complex magnetic configurations formed by a finite number of magnetic flux tubes, but as well in continuous 
magnetic configurations. Also, compared to all quantities which try to quantify the amount of magnetic stress present in a magnetic configuration, magnetic helicity has the unique property to be almost conserved in resistive MHD (on a timescale less than the global diffusion timescale, Matthaeus and Goldstein, 1982; Berger, 1984).

Its conservation defines a constraint to the magnetic field evolution; in particular, a stressed magnetic field with finite amount of helicity cannot relax to a potential field. This conservation property is linked to the cascade to large scales, while magnetic energy cascade to small scales where it is dissipated (Frisch et al., 1975; Alexakis et al., 2006). Thus, magnetic helicity is at the heart of several MHD theories: in laboratory plasma (e.g. relaxation toward a linear force-free field, Taylor, 1974), in the corona (e.g. its heating, Heyvaerts and Priest (1984), and the reconfiguration of the magnetic field, Zhang and Low (2003)), in the solar wind (e.g. development of MHD turbulence, Matthaeus et al., 1982). More recently, the conservation of magnetic helicity is identified as responsible of the dynamo saturation, the so-called $\alpha$ effect quenching (Brandenburg, 2001). These subjects have been extensively reviewed (Brown et al., 1999; Berger, 2003; Brandenburg and Subramanian, 2005) and the volume 32, Issue 10, of Advances in Space Research contains a series of papers on magnetic helicity.

During decades magnetic helicity remained mostly a theoretical subject with only crude attempts to relate it to observations. The reason is not that the potential applications were missing! For example, filaments and flares are known to be present in highly sheared magnetic configurations; S-shaped coronal loops are recurrently observed in the corona. Then, it is highly desirable to quantify better what sheared and twisted configurations mean. One of the main obstacles was the presence of the vector potential $\mathbf{A}(\mathbf{B}=\nabla \times \mathbf{A})$ in the definition of magnetic helicity, since it is far from any measurable quantity. Moreover, there is a freedom in the definition of $\mathbf{A}$. A gauge should be used to define $\mathbf{A}$ uniquely, but the magnetic helicity should be independent of this gauge to be physically meaningful. The gauge problem was solved theoretically two decades ago. Still the difficulty to compute magnetic helicity depends sharply on the gauge selected, and this best choice depends on the problem under analysis! Moreover, magnetic helicity may be expressed in a variety of ways. It is not simple to find the expression best adapted to available observations, being this still a topic of current research.

The main objective of this paper is to review the theoretical developments that have been applied to measurements of magnetic helicity in the solar atmosphere. After introducing different expressions for magnetic helicity, and comparing it to current helicity, Section 2 describes some practical computations and their applications to the corona and magnetic clouds. The theoretical expressions for the magnetic helicity rate (or total flux) through a surface have 
natural applications at the photospheric level (Section 3). It was realized that, using present data, not only the helicity rate, but also the flux density (or flux per unit surface) can be estimated. This is mostly limited by the measurement of photospheric flows. Since important progress has been recently made, this is the object of the separate Section 4. The implications of these advances for solar phenomena are summarized in Section 5. Finally, Section 6 outlines the fast progress achieved in the domain, as well as the probable next steps.

\section{Definition and measurements}

\subsection{In closed volumes}

The magnetic helicity of the field $\mathbf{B}(\mathbf{x})$ fully contained within a volume $\mathcal{V}$ (i.e. at any point of its boundary $\mathcal{S}$ the normal component $B_{n}=\mathbf{B} \cdot \hat{\mathbf{n}}$ vanishes) is (Elsasser, 1956):

$$
H^{\text {closed }}=\int_{\mathcal{V}} \mathbf{A} \cdot \mathbf{B} \mathrm{d}^{3} x
$$

where the vector potential $\mathbf{A}$ satisfies $\mathbf{B}=\nabla \times \mathbf{A}$. Since $B_{n}=0$ on $\mathcal{S}$, $H^{\text {closed }}$ is independent of the gauge selection for $\mathbf{A}$ (i.e., independent of the transformation $\mathbf{A} \rightarrow \mathbf{A}+\nabla \Phi$ where $\Phi$ is any single-valued derivable function of space and time). The vector potential $\mathbf{A}$ can be written in function of $\mathbf{B}$ within the Coulomb gauge, $\nabla \cdot \mathbf{A}=0$, just like the Biot-Savart integral for $\mathbf{B}$ :

$$
\mathbf{A}(\mathbf{x})=\frac{1}{4 \pi} \int_{\mathcal{V}} \mathbf{B}\left(\mathbf{x}^{\prime}\right) \times \frac{\left(\mathbf{x}-\mathbf{x}^{\prime}\right)}{\left|\mathbf{x}-\mathbf{x}^{\prime}\right|^{3}} \mathrm{~d}^{3} x^{\prime}
$$

Then the magnetic helicity can be written in function of $\mathbf{B}$ alone (Moffatt, 1969):

$$
H^{\text {closed }}=\frac{1}{4 \pi} \int_{\mathcal{V}} \int_{\mathcal{V}} \mathbf{B}(\mathbf{x}) \times \mathbf{B}\left(\mathbf{x}^{\prime}\right) \cdot \frac{\left(\mathbf{x}-\mathbf{x}^{\prime}\right)}{\left|\mathbf{x}-\mathbf{x}^{\prime}\right|^{3}} \mathrm{~d}^{3} x \mathrm{~d}^{3} x^{\prime} .
$$

Let us do the integration along and across the elementary flux tubes, called generically $a$ and $c$. Each tube contains an elementary magnetic flux $\mathrm{d} \Phi$. The element of integration $\mathrm{d}^{3} x$ is replaced by $\mathrm{d} S \mathrm{~d} l$, with $\mathbf{B} \mathrm{d} S=\hat{\mathbf{t}} \mathrm{d} \Phi$, and $\hat{\mathbf{t}}=\mathbf{B} / B$. Then, Equation (3) is rewritten as:

$$
H^{\text {closed }}=\int_{\Phi} \int_{\Phi} \mathcal{L}_{a, c}^{\text {closed }} \mathrm{d} \Phi_{a} \mathrm{~d} \Phi_{c}
$$




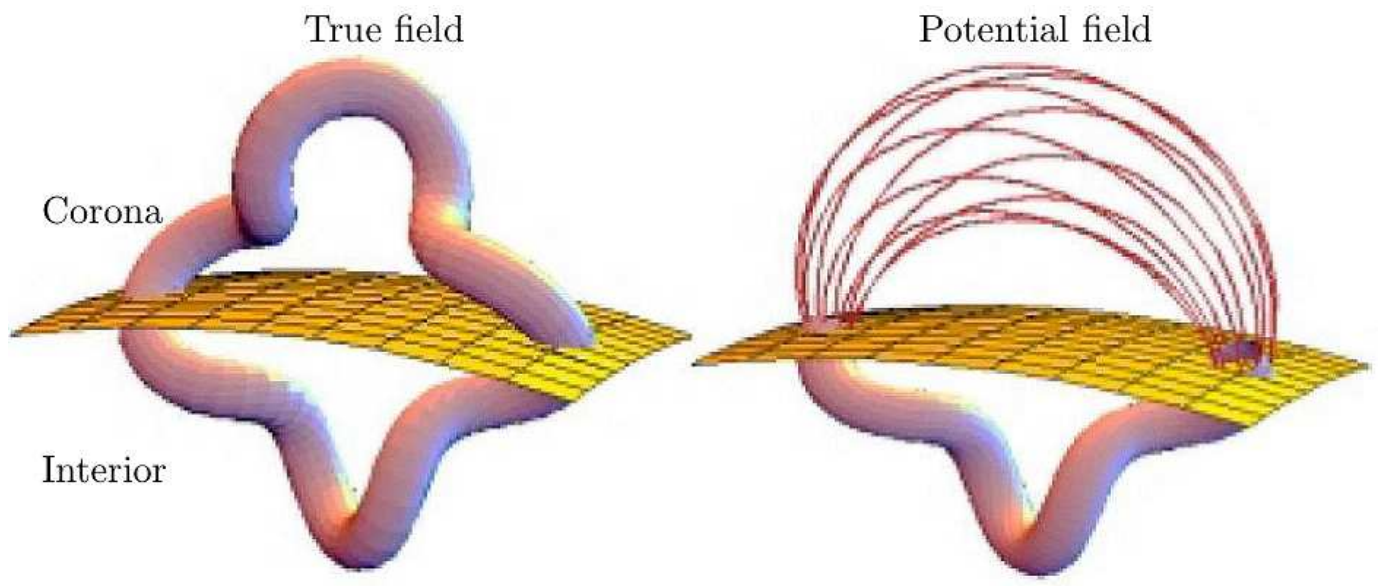

Fig. 1. Illustration of the computation of the relative helicity $H$ in an open volume (as the corona). $H$ is computed relative to the helicity of the potential field (Berger and Field, 1984). Both configurations are closed by the same magnetic field in the "interior" in order to use the original magnetic helicity definition for closed configurations, but $H$ is independent of the "interior" field selected (from Berger, 2003).

with $\Phi$ the total magnetic flux, and

$$
\mathcal{L}_{a, c}^{\text {closed }}=\frac{1}{4 \pi} \oint_{a} \oint_{c} \hat{\mathbf{t}}\left(\mathbf{x}_{a}\right) \times \hat{\mathbf{t}}\left(\mathbf{x}_{c}\right) \cdot \frac{\left(\mathbf{x}_{a}-\mathbf{x}_{c}\right)}{\left|\mathbf{x}_{a}-\mathbf{x}_{c}\right|^{3}} \mathrm{~d} l_{a} \mathrm{~d} l_{c} .
$$

$\mathcal{L}_{a, c}^{\text {closed }}$ is known as the Gauss linking number. It defines the number of times one flux tube turns around the other taking into account the orientations of the flux tubes. $\mathcal{L}_{a, c}^{\text {closed }}$ is a signed integer, invariant to deformations of the two flux tubes which do not involve one flux tube crossing the other. For two flux tubes which are not linked (which can be separated to infinitely large distance without crossing each other), one has $\mathcal{L}_{a, c}^{\text {closed }}=0$. Then, the magnetic helicity quantifies how much the elementary flux tubes of a configuration are inter-wounded.

\subsection{In open volumes}

In the case where $\mathcal{V}$ is part of the corona, we clearly have magnetic fluxes crossing its boundary $\mathcal{S}$, in particular for the part of $\mathcal{S}$ located at the photospheric level. Berger and Field (1984) have shown that for cases where $B_{n} \neq 0$ on some part of $\mathcal{S}$ one can define a relative magnetic helicity, $H$, by subtracting the helicity of a reference field $\mathbf{B}_{p}$ which has the same $B_{n}$ distribution on $\mathcal{S}$ and closing both magnetic configurations by the same magnetic field outside $\mathcal{S}$. This procedure is illustrated in Fig. 1. A general expression for $H$ is (Finn 
and Antonsen, 1985):

$$
H=\int_{\mathcal{V}}\left(\mathbf{A}+\mathbf{A}_{p}\right) \cdot\left(\mathbf{B}-\mathbf{B}_{p}\right) \mathrm{d}^{3} x
$$

It is independent of the gauge selected for $\mathbf{A}$ and $\mathbf{A}_{p}$ since any change of gauge adds a term of the form $\nabla \Phi$ to $\mathbf{A}+\mathbf{A}_{p}$, and the extra integral vanishes because $\nabla \cdot \mathbf{B}=\nabla \cdot \mathbf{B}_{p}=0$ and $B_{n}=B_{p, n}$ on $\mathcal{S}$. Taking the boundary condition for the tangential components:

$$
\mathbf{A}_{t}=\mathbf{A}_{p, t} \text { on } \mathcal{S}
$$

satisfies $B_{n}=B_{p, n}$ on $\mathcal{S}$ and permits the re-expression of Eq. (6) as a difference of helicities:

$$
H=\int_{\mathcal{V}} \mathbf{A} \cdot \mathbf{B} \mathrm{d}^{3} x-\int_{\mathcal{V}} \mathbf{A}_{p} \cdot \mathbf{B}_{p} \mathrm{~d}^{3} x
$$

As the potential field is the simplest field, with the lowest magnetic energy, for a given distribution of $B_{n}$ on $\mathcal{S}$, it is usually taken as the reference field $\mathbf{B}_{p}$.

While Eqs. $(6,8)$ are gauge-invariant, some gauges are easier to use than others! For the potential field $\mathbf{B}_{p}$, the classical gauge conditions are the following ones (Barnes, 1988; Berger, 1988):

$$
\begin{aligned}
\mathbf{A}_{p} \cdot \hat{n}=0 & \text { on } \mathcal{S}, \\
\nabla \cdot \mathbf{A}_{p}=0 & \text { in } \mathcal{V} .
\end{aligned}
$$

First, with $\nabla \times \mathbf{A}_{p}=\mathbf{B}_{p}$, these conditions define $\mathbf{A}_{p}$ uniquely. Second, an explicit expression of $\mathbf{A}_{p}$ exists in classical geometries (this is important for helicity rate computation, see Section 3). Finally, the helicity of $\mathbf{B}_{p}$ vanishes (since $\mathbf{B}_{p}$ can be written as a gradient of a scalar potential), so that $H$ has the same expression as $H^{\text {closed: }}$

$$
H=\int_{\mathcal{V}} \mathbf{A} \cdot \mathbf{B} \mathrm{d}^{3} x
$$

Different gauges can be used for $\mathbf{A}$, and this freedom can be used to simplify the computation of A. Berger (1988) used:

$$
\nabla \cdot \mathbf{A}=0 \text { in } \mathcal{V}
$$


Eq. (12), together with $\nabla \times \mathbf{A}=\mathbf{B}$ and Eq. (7), defines $\mathbf{A}$ uniquely. This implies solving partial differential equations in $\mathcal{V}$, which is difficult unless symmetries are present.

Hornig (2006) proposed a definition of magnetic helicity which does not need a reference magnetic field. The definition is based on Eq. (11) and:

$$
\nabla_{t} \cdot \mathbf{A}_{t}=0 \text { on } \mathcal{S}
$$

where the subscript $t$ means the tangential components (parallel to $\mathcal{S}$ ). This boundary condition restricts the gauge freedom, $\mathbf{A} \rightarrow \mathbf{A}+\nabla \Phi$, to $\Phi$ being constant on $\mathcal{S}$, then all possible vector potentials have the same tangential component on $\mathcal{S}$ (being Eq. (7) a particular case). This implies that $H$ is gauge invariant. Hornig's helicity definition gives the same value as the relative helicity (Eq. 6) when the boundary is a plane or a sphere. Moreover, it has all the expected properties of magnetic helicity, and it has explicitly the same definition for $\mathbf{B}$ in open and closed volumes. However, it is not unique for a multiply connected volume (a case not considered in this review), and its properties need to be further explored.

Low (2006) decomposed any field $\mathbf{B}$ into a pair of globally untwisted fields, $\mathbf{B}=\mathbf{B}_{t}+\mathbf{B}_{s}$, and gave some explicit possible decompositions. The untwisted condition permits to write $\mathbf{B}_{t}$ with Euler potentials $\left(\mathbf{B}_{t}=\nabla \xi \times \nabla \zeta\right.$, and $\xi$ and $\zeta$ are constant along field lines of $\mathbf{B}_{t}$ ). Then, he showed that magnetic helicity can be decomposed as the integral of the so-called primitive helicity, $\Phi_{s}(\xi, \zeta) \mathrm{d} \Phi_{t}$, where $\Phi_{s}$ is the flux of $\mathbf{B}_{s}$ encircled by the elementary flux $\mathrm{d} \Phi_{t}$ surrounding the field line $(\xi, \zeta)$ of $\mathbf{B}_{t}$. This defines an infinite number of ideal invariants for any continuous magnetic configurations. They are equivalent to the frozen-in condition. Since only magnetic fluxes are present in the definition, there is no gauge involved. Moreover, the total magnetic helicity is independent of the decomposition selected and there is no difference between open and closed configurations. Finally, it is worth to note that the primitive helicity is

not defined by the elementary flux tubes of $\mathbf{B}$, but by those of $\mathbf{B}_{t}$ and that a large freedom is present in its selection.

\subsection{Comparison to current helicity}

The current helicity is defined in a similar way as the magnetic helicity:

$$
H_{c}=\int_{\mathcal{V}} \mathbf{B} \cdot \mathbf{j} \mathrm{d}^{3} x
$$


with $\mu_{0} \mathbf{j}=\nabla \times \mathbf{B}$. It measures how much the $\nabla \times \mathbf{B}$ is along $\mathbf{B}$, so how much the magnetic field is locally twisted. More precisely, $H_{c}$ measures how much the elementary current channels are inter-wounded, as in the case of magnetic helicity for flux tubes. Behind this similarity there are important differences. First, there is no gauge freedom with $H_{c}(\nabla \cdot \mathbf{B}=0)$. Second, while $H$ is a conserved MHD quantity, this is not the case for $H_{c}$. Finally, even in the case of force-free fields $(\mathbf{j}=\alpha \mathbf{B})$ where current channels and flux tubes coincide, $H_{c}$ and $H$ are generally not simply related (the distribution of $\alpha$ is also a key ingredient). It is usually assumed that $H_{c}$ and $H$ have the same sign, but even this is so far not generically demonstrated to my knowledge. For example, a clockwise vortex applied locally inside one magnetic polarity of an initial potential field wound all the field lines in the same direction giving all a positive contribution to $H$. However because the vortex is limited in space, the induced currents in the centre (direct currents) are surrounding by currents of opposite direction (return currents). Direct and reverse currents have an opposite contribution to $H_{c}$. Generally, but not necessarily always, the direct currents have a dominant contribution to $H_{c}$, so that $H_{c}$ and $H$ have the same sign.

The dissipation of magnetic helicity is directly linked to $H_{c}$ :

$$
\left.\frac{\mathrm{d} H}{\mathrm{~d} t}\right|_{\text {dis. }}=-2 \int_{\mathcal{V}} \mathbf{E} \cdot \mathbf{B} \mathrm{d}^{3} x=-2 H_{c} / \sigma,
$$

where $\sigma$ is the resistivity of the plasma (assumed to be uniform), and the simple Ohm's law, $\mathbf{j}=\sigma \mathbf{E}$, is used to derive the right hand term of the above equation. This relates only the dissipation of $H$ to $H_{c}$, not the helicities present at a given time in the magnetic configuration. Moreover for the solar plasma, the right hand term in Eq. (15) is very small (giving the conserved property of $H$, Berger, 1984), and this equation is of no use to relate both helicities.

Using photospheric vector magnetograms all three components of $\mathbf{B}$ are available, but only the vertical component of $\mathbf{j}, j_{z}$, can be computed (via the horizontal $x, y$ derivatives of $\mathbf{B}$ ). The three components of $\mathbf{j}$ can be derived only if a force-free field is assumed. This is justified only if the magnetograms are taken with a spectral line formed high enough in the atmosphere (Metcalf et al., 1995). Thus, most studies have derived maps of $B_{z} \cdot j_{z}$ at the photospheric level. The scatter of this quantity in an active region (AR) is usually large, with values of both signs intermixed. This mixed pattern has various origins: the important noise in the transverse magnetic field, the amplification of the noise by the spatial derivatives (to compute the current density), the lack of spatial resolution to identify magnetic flux tubes, the presence of return currents, and, possibly, the presence of flux tubes with opposite $H_{c}$. Still a dominant sign is found usually for $H_{c}$ in ARs, with a latitude depen- 
dence independent of the solar cycle (e.g. Abramenko et al., 1996; Bao and Zhang, 1998). Typically $\approx 80 \%$ of the ARs in the northern (southern) hemisphere have $H_{c}<0(>0)$. Another approach is to fit a linear force-free field, $\mathbf{j}=\alpha \mathbf{B}$, to the photospheric vector magnetic field to compute a single value $\alpha_{\text {best }}$ (Pevtsov et al., 1995). The magnitude of the hemispherical dominance is less strong, typically $\approx 70 \%$ of the ARs in the northern (southern) hemisphere have $\alpha_{\text {best }}<0(>0)$. These hemispherical rules are confirmed by further studies, while the importance of the dominance depends on the data set analyzed and the proxy of the helicity used (Bao et al., 2000; Hagino and Sakurai, 2004).

\subsection{Practical computation in the corona}

Magnetic helicity is a multi-faceted quantity. Some of the most usual ways to compute it are given below, but this is not a restrictive list! In the application to the solar case, $\mathcal{V}$ is a part of the coronal volume (corresponding e.g. to the magnetic field of an active region). Magnetic field measurements are typically available only at the photospheric level, so on a fraction of $\mathcal{S}$, called $\mathcal{S}_{p}$. In what follows, $\mathcal{S}_{p}$ is supposed to be planar (equivalent expressions could be derived for $\mathcal{S}_{p}$ located on a sphere). On the remaining coronal part of $\mathcal{S}$, $\mathcal{S}_{c}\left(\mathcal{S}=\mathcal{S}_{p}+\mathcal{S}_{c}\right)$, no direct observations are available. $\mathcal{V}$ is generally taken large enough so that the results are not significantly affected by the boundary conditions set on $\mathcal{S}_{c}$.

Amari et al. (2003a,b) used Eqs. $(7,10,12)$, and $\nabla_{t} \cdot \mathbf{A}_{p, t}=0$ on $\mathcal{S}_{p}$ rather than Eq. (9). But, since $\mathbf{B}_{p}$ is a potential field this also implies $A_{p, n}=0$ (on $\mathcal{S}_{p}$ but also in $\mathcal{V}$ ), so in fact Amari et al. used the same boundary conditions as Berger (1988). Then, they solved $\nabla \times \mathbf{A}=\mathbf{B}$ in the $3 \mathrm{D}$ volume $\mathcal{V}$ with this gauge and boundary conditions.

A practical way to compute the magnetic helicity, in particular for analytical computations, is to split the field $\mathbf{B}$ in two: $\mathbf{B}=\mathbf{B}_{\text {closed }}+\mathbf{B}_{\text {open }}$, where $\mathbf{B}_{\text {closed }}$ is fully contained in $\mathcal{V}\left(B_{n \text {,closed }}=0\right.$ on $\left.\mathcal{S}\right)$, and $\mathbf{B}_{\text {open }}$ has the same distribution as $\mathbf{B}$ on $\mathcal{S}\left(B_{n, \text { open }}=B_{n}\right)$. The simplest choice is usually to take $\mathbf{B}_{\text {open }}$ as the potential field $\mathbf{B}_{p}$. Moreover, with Eqs. $(9,10)$ one has $\int_{\mathcal{V}} \mathbf{A}_{p} \cdot \mathbf{B}_{p} \mathrm{~d}^{3} x=0$, and the relative helicity is reduced to (Berger, 2003):

$$
H=\int_{\mathcal{V}} \mathbf{A}_{\text {closed }} \cdot \mathbf{B}_{\text {closed }} \mathrm{d}^{3} x+2 \int_{\mathcal{V}} \mathbf{A}_{p} \cdot \mathbf{B}_{\text {closed }} \mathrm{d}^{3} x
$$

In this formulation, the helicity is the sum of the self helicity of the closed field and the mutual helicity of the closed and open fields. 
(a) $H=\left(\mathcal{L}_{a, c}^{\text {arch }}-2\right) \Phi_{a} \Phi_{c}$

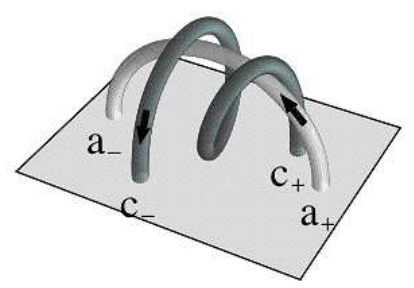

(b) $H=-\mathcal{L}_{a, c}^{\mathrm{arch}} \Phi_{a} \Phi_{c}$

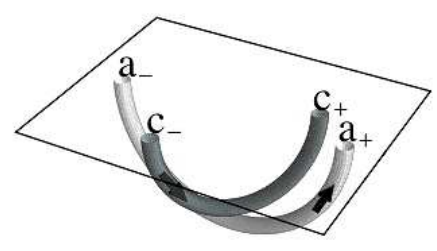

(c) $H=-2 \Phi_{a} \Phi_{c}$

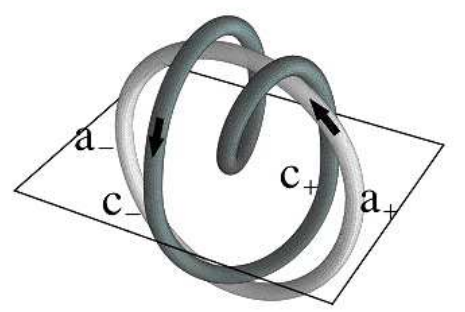

Fig. 2. Illustration with a pair of flux tubes of the magnetic helicity computation directly from the flux tube properties (the general expression involves a double integration over the pairs of elementary flux tubes: Eq. (18). The helicity of the open configuration (a) is obtained by closing it with the configuration (b) (the mirror image, through the planar boundary $\mathcal{S}_{p}$, of an arch-like configuration having the same footpoint locations as (a)). The sum of (a) and (b) gives the closed configuration (c). The mutual helicity of (b) depends only on the position of the footpoints $\left(a_{-}, a_{+}, c_{-}, c_{+}\right)$and that of (c) is the Gauss linkage number (Eq. 5). The magnetic helicity of (a) is the difference between that of (c) and (b) (from Démoulin et al., 2006).

For numerical computations of the helicity from a known field $\mathbf{B}$ in $\mathcal{V}$, the clever gauge selection of DeVore (2000) is probably the most practical one. $\mathbf{A}_{p}$ is computed with Eqs. $(9,10)$ and Eq. (7) is used, so that the helicity is simply given by Eq. (11). Next, Eq. (12) is replaced by $A_{z}=0$, where $z$ is the cartesian coordinate orthogonal to $\mathcal{S}_{p}$. This gauge selection greatly simplifies the computation of $\mathbf{A}$ from $\mathbf{B}$ in $\mathcal{V}$ : rather than solving partial differential equations in $\mathcal{V}$, only simple line integrations are required:

$$
\mathbf{A}(x, y, z)=\mathbf{A}_{p}(x, y, 0)-\hat{\mathbf{z}} \times \int_{0}^{z} \mathbf{B}\left(x, y, z^{\prime}\right) \mathrm{d} z^{\prime}
$$

In this approach $\mathbf{A}_{p}$ needs to be computed only on the boundary $\mathcal{S}_{p}$, while $\mathbf{A}$ is computed in the volume $\mathcal{V}$ with Eq. (17).

The use of $\mathbf{A}$ hides the physics of magnetic helicity (how the magnetic flux tubes are interlaced). Démoulin et al. (2006) showed that the helicity of open configurations can be computed, like for closed ones, as a summation over the elementary flux tubes forming the configuration (Fig. 2). They computed the helicity by successively bringing elementary flux tubes to build up the magnetic configuration, and count the helicity injected by tracking the footpoint motions on $\mathcal{S}_{p}$. The magnetic helicity is

$$
H=\int_{\Phi} \int_{\Phi}\left(\mathcal{L}_{a, c}^{\text {closed }}+\mathcal{L}_{a, c}^{\text {arch }}\right) \mathrm{d} \Phi_{a} \mathrm{~d} \Phi_{c}
$$


where the double integration is done over all pairs of elementary flux tubes "a" and "c" with magnetic fluxes $\mathrm{d} \Phi_{a}$ and $\mathrm{d} \Phi_{c}$. The first contribution $\mathcal{L}_{a, c}^{\text {closed }}$, defined by Eq. (5), counts how much the closed flux tubes wind around each other (Fig. 2c). $\mathcal{L}_{a, c}^{\text {closed }}$ is a signed integer. The second contribution, $\mathcal{L}_{a, c}^{\text {arch }}$, depends only on the position on $\mathcal{S}_{p}$ of the footpoints, called $a_{-}, a_{+}, c_{-}$and $c_{+} \cdot \mathcal{L}_{a, c}^{\text {arch }}$ is a real number in the open interval $(-1,1)$, and it vanishes if the flux tubes do not cross $\mathcal{S}_{p}$. More precisely, $\mathcal{L}_{a, c}^{\text {arch }}$ is defined with the interior angles $\alpha_{a_{+}}, \alpha_{a_{-}}$(or $\alpha_{c_{+}}, \alpha_{c_{-}}$), given by the relative positions of the footpoints, as follow. The index of $\alpha$ indicates which foot point is located at the vertex of the angle. For example, $\alpha_{a_{+}}$defined the viewing angle between $c_{-}$and $c_{+}$ as seen from $a_{+}$. Then we define the two functions: $\mathcal{L}_{\hat{\mathbf{a}}, c}^{\mathrm{arch}}=\left(\alpha_{a_{+}}+\alpha_{a_{-}}\right) /(2 \pi)$ and $\mathcal{L}_{a, \hat{\mathbf{c}}}^{\text {arch }}=\left(\alpha_{c_{+}}+\alpha_{c_{-}}\right) /(2 \pi)$, where the index $\hat{\mathbf{a}}(\hat{\mathbf{c}})$ indicates that the angles $\alpha$ are centered on the foot points of flux tube "a" ("c"), respectively. Then, Démoulin et al. (2006) demonstrated that $\mathcal{L}_{a, c}^{\text {arch }}$ is given by:

$$
\begin{aligned}
\mathcal{L}_{a, c}^{\operatorname{arch}} & =\mathcal{L}_{\hat{\mathrm{a}}, c}^{\mathrm{arch}} & & \text { if "a" above "c" } \\
& =\mathcal{L}_{a, \hat{\mathbf{c}}}^{\mathrm{arch}} & & \text { if "c" above "a" } \\
& =\mathcal{L}_{\hat{\mathbf{a}}, c}^{\mathrm{arch}}=\mathcal{L}_{a, \hat{\mathbf{c}}}^{\operatorname{arch}} & & \text { otherwise }
\end{aligned}
$$

where flux tube "a" is called "above" flux tube "c" when the closed curve defined by the elementary flux tube "a" and the segment $a_{-} a_{+}$encircles flux tube "c".

Equation (18) sets closed and open configurations on an equal level: in both cases we do not need to compute the reference potential field (although the results are always equivalent to Eq. (6). Moreover, the helicity of closed configurations can be seen as a particular case of the helicity of open configurations: the contribution of the footpoints simply disappears (the closed configuration can be formed by bringing the footpoints of the associated open configuration together), then Eq. (18) reduces to Eq. (4). On the other hand, we can close any open configuration by a field outside $\mathcal{V}$ which is formed only of arch-like flux tubes, and so we can associate to any open configuration a closed configuration. Next, the derived method does not rely on computing a vector potential field, but rather uses only observable quantities: the normal field component at the boundary and the flux tube linkages (connectivities and how each pair winds around each other). Then, it has natural applications for computing the coronal helicity directly from a photospheric magnetogram and an ensemble of observed coronal loops. 


\subsection{Measurements in the corona}

So far, the direct determination of the magnetic helicity $H$ contained in the corona has used the computation of the magnetic field from a photospheric magnetogram (called extrapolation). An indirect determination was realized via the temporal summation of the helicity rate (Section 3 ).

For a linear force-free field, $\mathbf{j}=\alpha \mathbf{B}$ with $\alpha$ a given constant, the general expression of magnetic helicity was given by Berger (1985) as a summation over the Fourier modes of the magnetogram. When $\alpha$ exceeds a certain critical value, which depends on the horizontal size of the integration box, $H$ has unphysical large values, then Démoulin et al. (2002a) proposed to linearized Berger's expression in function of $\alpha$, giving:

$$
H=\alpha \sum_{n_{x}=0}^{N_{x}} \sum_{n_{y}=0}^{N_{y}}\left|\widetilde{B}_{n_{x}, n_{y}}^{2}\right| /\left(k_{x}^{2}+k_{y}^{2}\right)^{3 / 2},
$$

where the mode $n_{x}=n_{y}=0$ (uniform field) has no contribution, and $k_{x}=$ $2 \pi n_{x} / L, k_{y}=2 \pi n_{y} / L, L$ being the horizontal extension of the computational box. It is worth noting that this equation has been corrected from the typos present in the original paper. However, the typos were not present in the computations reported.

The value of $\alpha$ is determined by minimizing the distance between the observed coronal loops and the computed field lines. Applying this approach to the longterm evolution of two active regions (ARs), which have a typical magnetic flux $\Phi \approx 1.5 \times 10^{22} \mathrm{Mx}$, Démoulin et al. (2002a) and Green et al. (2002) found a maximum helicity stored at a given time in the corona $H \approx 40 \times 10^{42} \mathrm{Mx}^{2}$ (when corrected for the flux underestimation by MDI, Berger and Lites, 2003). This is a relatively low amount: a flux tube uniformly twisted with $n$ turns has an helicity $n \Phi^{2}$, so $H / \Phi^{2}$ has a maximum of about 0.2 turn for both ARs. For a tiny isolated magnetic bipole, $\Phi \approx 2 \times 10^{20} \mathrm{Mx}$, Mandrini et al. (2005) obtained a maximum $H \approx 7 \times 10^{39} \mathrm{Mx}^{2}$, so $H / \Phi^{2} \approx 0.18$, a value similar to the value obtained for large ARs. At that point, it is worth to note that the general property of a linear force free extrapolation is that the shorter (resp. longer) field lines are less (resp. more) sheared than the observed coronal loops (a consequence of the helicity cascade to large scales in this minimum energy field for a given magnetic helicity). Since $\alpha$ is mostly determined by large scale loops, Eq. (20) is expected to underestimate the amount of helicity, an expectation confirmed by Nindos and Andrews (2004) who compared the estimation of the extrapolation to the temporal summation of the helicity rate.

With a non-linear force-free field, $\alpha$ is constant along each field lines, but otherwise variable in space. This extra information is derived from photospheric 

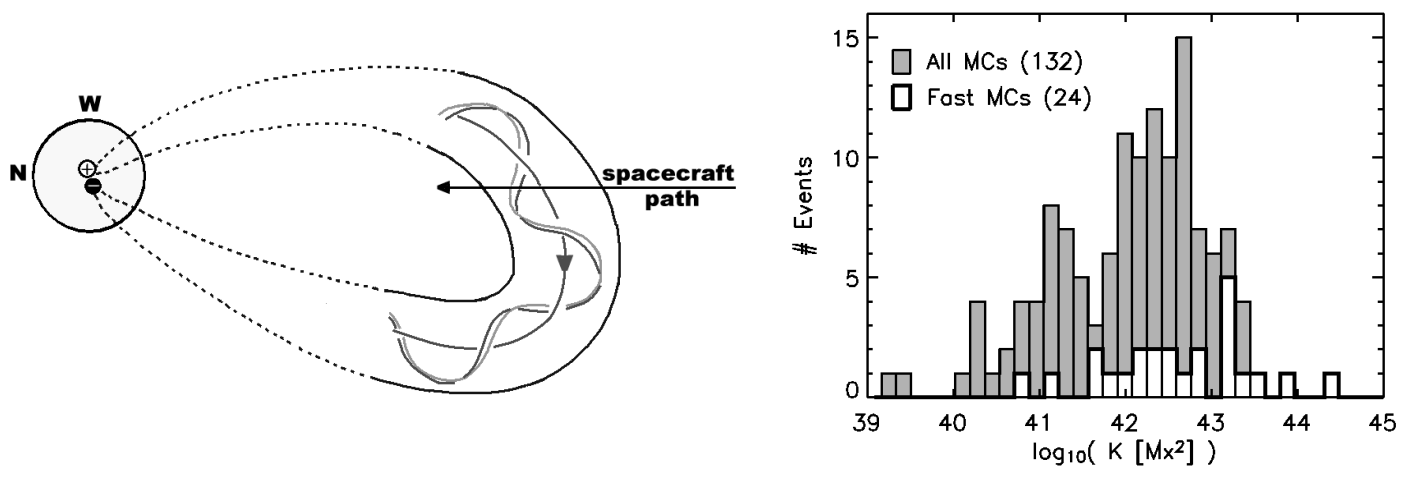

Fig. 3. Left panel: Schematic global view of a magnetic cloud (MC) and its solar source region. The twisted flux rope of the MC is represented by few field lines (continuous lines). The dashed lines indicate the possible continuation of the rope to the Sun at the time the MC crosses the spacecraft (from Mandrini et al., 2005). Right panel: Histogram of the logarithm of the magnetic helicity found in MCs computed with a linear force free model fitting the data. The histogram of fast events (velocity $>500 \mathrm{~km} . \mathrm{s}^{-1}$ ) is over plotted in white (from Lynch et al., 2005).

vector magnetograms. The computation of the magnetic helicity is done numerically from the extrapolated magnetic field (Section 2.4). Régnier et al. (2005) found $H \approx-4.2 \times 10^{42} \mathrm{Mx}^{2}$ for an AR having $\Phi \approx 1.3 \times 10^{22} \mathrm{Mx}$. This is even a lower helicity contain than above: $|H| / \Phi^{2} \approx 0.02$. Even more surprising the helicity sign is opposite to what is deduced from the curling of the $H_{\alpha}$ fibrils and from the clockwise rotation of the main sunspot. In this active region, Moon et al. (2002b) and Nindos et al. (2003) deduced independently a positive helicity rate (providing an helicity $\approx 40 \times 10^{42} \mathrm{Mx}^{2}$ in 6 days) The above controversy most probably comes from present limitations of vector magnetograms which are too noisy and biased to determine $\alpha$ accurately enough. The extrapolation with non-linear force-free fields is also presently an active domain of research. The estimation of the coronal helicity with a non-linear force-free field awaits the next generation of vector magnetograms and extrapolation codes.

\subsection{Measurements in magnetic clouds}

Coronal mass ejections (CMEs) appear in the interplanetary medium as interplanetary CMEs (ICMEs). A subset of these ICMEs, known as magnetic clouds (MCs), is characterized by enhanced magnetic field strength with respect to ambient values, a smooth and large rotation of the magnetic field vector, and low proton temperature (Burlaga, 1995). Inside the MC, the magnetic field lines form a helical structure (the so called magnetic flux rope), therefore, an MC carries a large amount of magnetic helicity (Fig. 3). This magnetic configuration is very different from the typical solar wind, which is 
basically the Parker's spiral.

There is increasing observational evidence that the flux-rope orientation, the magnetic field direction, as well as, the helicity sign in magnetic clouds matches with their source regions (see e.g., Bothmer and Schwenn, 1994; Rust, 1994; Marubashi, 1997; Yurchyshyn et al., 2001). In order to quantify this link, through estimation of magnetic fluxes and helicities, a magnetic model is needed, since data are available only along the spacecraft trajectory across the MC. The most widely used models have a cylindrical geometry: $\mathbf{B}(\mathbf{r})=$ $B_{\varphi}(r) \hat{\varphi}+B_{z}(r) \hat{\mathbf{z}}$, where $r$ is the radial coordinate, $z$ is along the flux rope axis and $\varphi$ indicates the azimuthal component (see Dasso et al., 2005, for a review). With the definitions of Section 2.2, we find the helicity per unit length:

$$
\frac{H}{L}=4 \pi \int_{0}^{R} A_{\varphi}(r) B_{\varphi}(r) r d r
$$

where $R$ is the radius of the $\mathrm{MC}$ and the azimuthal component of the vector potential $A_{\varphi}(r)=\left(\int_{0}^{r} r^{\prime} B_{z}\left(r^{\prime}\right) d r^{\prime}\right) / r$.

Within the frame co-moving with the $\mathrm{MC}$, the inertial term is usually small compared to the magnetic pressure term in the momentum equation. Moreover the plasma $\beta$ (ratio of plasma to magnetic pressure) is usually low in MCs (around 0.1), then force free models are frequently used (e.g., Farrugia et al., 1999; Lepping et al., 1990). Models including plasma pressure have also being proposed (Cid et al., 2002; Hidalgo et al., 2002). The free parameters of the models are derived by a least square fit to the data. From the $18 \mathrm{MCs}$ analyzed by Lepping et al. (1990) with a linear force free field, DeVore (2000) found a mean helicity of $\approx 2 \times 10^{42} \mathrm{Mx}^{2}$ assuming a MC length of $0.5 \mathrm{AU}$. A similar mean value is found from the $23 \mathrm{MCs}$ analyzed in a similar way by Zhao et al. (2001). However, individual MC can contain a magnetic helicity very different from this mean. This is best seen on the large sample analyzed by Lynch et al. (2005) as shown in Fig. 3.

The estimation of magnetic helicity in MCs is subjected to several uncertainties.

- First, the helicity previously derived is model dependent. Within the cylindrical hypothesis, force-free and non force-free models give helicities which differ by up to $30 \%$ (Dasso et al., 2003, 2006). However, this variation remains small compared to the variation of helicity computed between different MCs (Gulisano et al., 2005). Moreover, within the cylindrical hypothesis, Dasso et al. (2006) showed that the magnetic helicity can be estimated directly from the magnetic data.

- Second, the MC boundaries can be defined by several criteria, which do not always agree. This introduces an uncertainty on the magnetic helicity which 
can be comparable to the uncertainty obtained with different models (Dasso et al., 2006). Moreover, in few cases there are disagreements in the detection of MCs (Russell and Shinde, 2005).

- Third, at least part of the MCs have not circular cross section (Riley et al., 2003). Some of the cylindrical models have been extended to incorporate an elliptical cross section (Vandas and Romashets, 2003; Hidalgo et al., 2002). Another approach is a magnetic extrapolation from the data using the force balance (Hu and Sonnerup, 2002). In this approach, the observed plasma pressure is taken into account and no hypothesis is done on the cross-section shape. However, a ill-posed problem has to be solved, implying that the results are sensitive to the regularization method used.

- Finally, the distribution of the twist along the flux rope, as well as its length are generally not known. The in-situ detection of suprathermal electrons and/or protons, moving along the magnetic field, is generally interpreted as an evidence that magnetic field lines are still attached to the Sun (Gosling et al., 1987). So far, only in one case the length of field lines could be determined precisely from impulsive electron events and solar type III radio bursts (Larson et al., 1997).

So magnetic helicity measurements in MCs is still in its infancy, and much progresses are expected from the STEREO mission.

\section{$3 \quad$ Flux of magnetic helicity}

\subsection{Definition}

The changes of the magnetic helicity content within the volume $\mathcal{V}$ are due to both fluxes across $\mathcal{S}\left(\mathcal{S}_{p}+\mathcal{S}_{c}\right)$ and dissipation within $\mathcal{V}$. In the application to the solar case, $\mathcal{V}, \mathcal{S}_{p}, \mathcal{S}_{c}$ are a part of the coronal volume, its photospheric and coronal boundaries, respectively. $\mathcal{S}_{p}$ is supposed to be planar, and no direct measurement is available on $\mathcal{S}_{c}$, as in Section 2.4. The rate (or total flux) of magnetic helicity through $\mathcal{S}_{c}$ can be estimated only indirectly from the CMEs leaving $\mathcal{V}$ and detected in the interplanetary space as MCs (Section 2.6).

The evolution of $H$ inside $\mathcal{V}$ is (Berger and Field, 1984):

$$
\begin{aligned}
\frac{\mathrm{d} H}{\mathrm{~d} t} & =2 \int_{\mathcal{S}} \mathbf{A}_{p} \times(\mathbf{v} \times \mathbf{B}) \cdot \hat{n} d S+\left.\frac{\mathrm{d} H}{\mathrm{~d} t}\right|_{\text {dis. }} \\
& =\left.\frac{\mathrm{d} H}{\mathrm{~d} t}\right|_{t}+\left.\frac{\mathrm{d} H}{\mathrm{~d} t}\right|_{n}+\left.\frac{\mathrm{d} H}{\mathrm{~d} t}\right|_{\mathcal{S}_{c}}+\left.\frac{\mathrm{d} H}{\mathrm{~d} t}\right|_{\text {dis. }},
\end{aligned}
$$


where $\mathbf{v}$ is the plasma velocity and $\mathbf{A}_{p}$ satisfies Eqs. $(9,10)$. In the last line, the tangential (shearing/twisting) and normal (emergence) helicity rates across $\mathcal{S}_{p}$ are separated:

$$
\begin{aligned}
& \left.\frac{\mathrm{d} H}{\mathrm{~d} t}\right|_{t}=-2 \int_{\mathcal{S}_{p}}\left(\mathbf{A}_{p} \cdot \mathbf{v}_{t}\right) B_{n} \mathrm{~d} \mathcal{S}, \\
& \left.\frac{\mathrm{d} H}{\mathrm{~d} t}\right|_{n}=+2 \int_{\mathcal{S}_{p}}\left(\mathbf{A}_{p} \cdot \mathbf{B}_{t}\right) v_{n} \mathrm{~d} \mathcal{S} .
\end{aligned}
$$

In pioneering works, since only horizontal velocities were deduced from the temporal evolution of $B_{n}$, it was supposed that only the tangential term could be derived (e.g. Chae et al., 2001; Nindos and Zhang, 2002; Moon et al., $2002 \mathrm{~b}$ ). However, the magnetograms of $B_{n}$ allow us to follow the photospheric intersection of the magnetic flux-tubes but not the evolution of the plasma (even if the plasma is frozen in the field, the two velocities are usually different). From the observed magnetic evolution one can estimate the flux-tube motion, $\mathbf{u}$, parallel to $\mathcal{S}$, not the plasma motion, $\mathbf{v}$. They are related by:

$$
\mathbf{u}=\mathbf{v}_{t}-\frac{v_{n}}{B_{n}} \mathbf{B}_{t}
$$

and the photospheric helicity rate simplifies to

$$
\left.\frac{\mathrm{d} H}{\mathrm{~d} t}\right|_{\mathcal{S}_{p}}=-2 \int_{\mathcal{S}_{p}}\left(\mathbf{A}_{p} \cdot \mathbf{u}\right) B_{n} \mathrm{~d} \mathcal{S},
$$

allowing the full helicity rate to be deduced from a time series of longitudinal magnetograms (Démoulin and Berger, 2003).

The helicity dissipation, $d H /\left.d t\right|_{\text {dis. }}$, is negligible in all the processes occurring in the corona, including the non-ideal ones (Berger, 1984). The dissipation time scale of magnetic helicity is the global diffusion time scale. For example, with a classical resistivity, Berger (1984) found a minimum helicity dissipation time of the order of $10^{5}$ years in a typical coronal loop! An upper bound on the helicity dissipation scales as $R_{M}^{-1 / 2}$, where $R_{M}$ is the magnetic Reynolds number, but the dissipation can be even smaller at high $R_{M}$ since it scales as $R_{M}^{-2}$ in magnetic reconnection events (Freedman and Berger, 1993).

\subsection{Magnitude of photospheric rates}

Wang (1996) gave the first order of magnitude of the helicity rate expected 

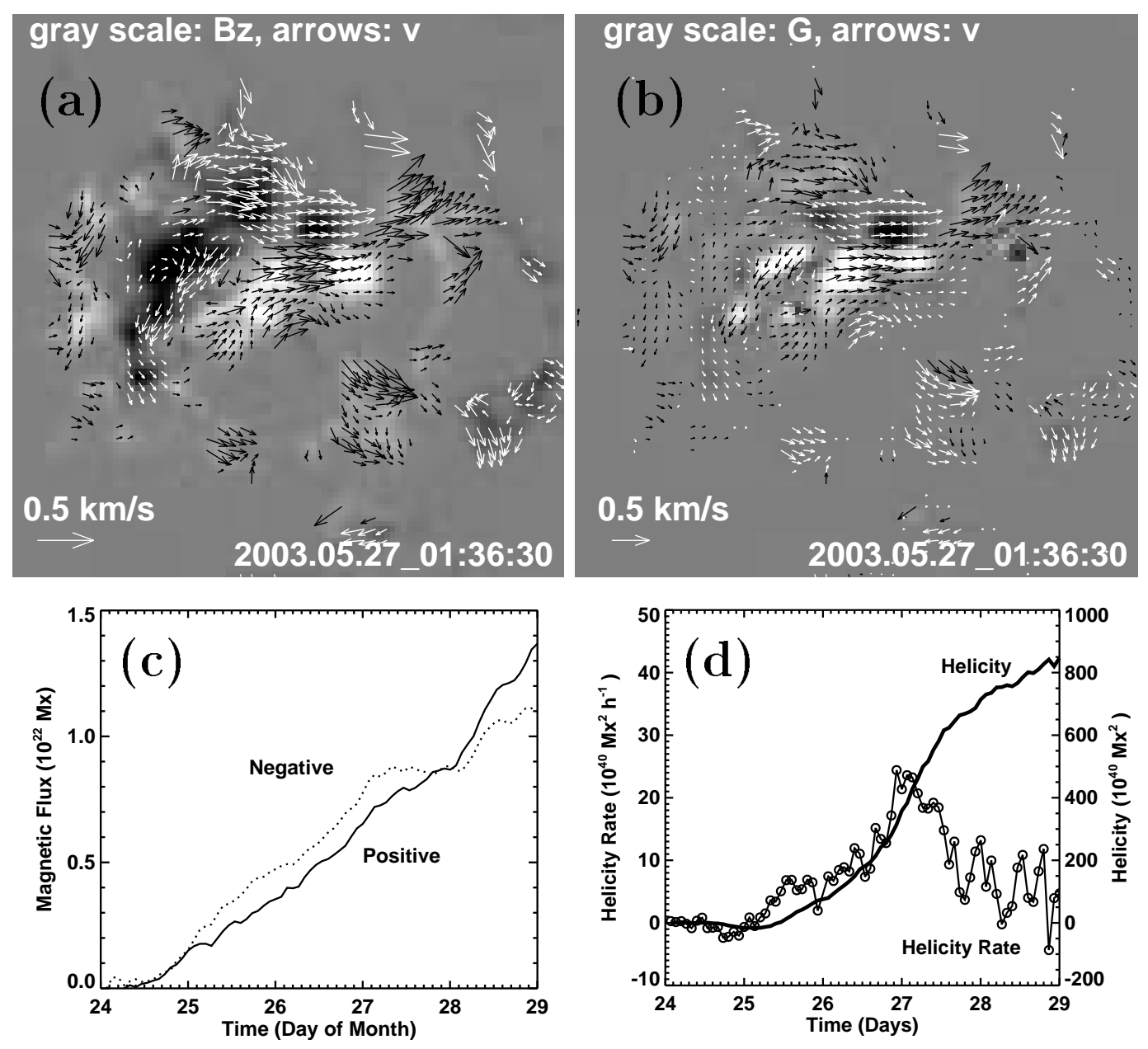

Fig. 4. Photospheric emergence of an AR (NOAA 10365). (a) Photospheric velocity (arrows) deduced from LCT (Section 4.1) superposed on the map of the normal field component (grey scale). (b) Same velocity field superpose on the flux density $G_{A}$ (Eq. 28). (c) Magnetic flux evolution of both polarities. (d) Helicity rate and the accumulate amount of magnetic helicity (from Chae et al., 2004).

in an $\mathrm{AR} \approx 700 \times 10^{40} \mathrm{Mx}^{2} / \mathrm{h}$ assuming an average photospheric velocity of $0.5 \mathrm{~km} . \mathrm{s}^{-1}$. More quantitative estimations use velocity maps derived by one of the techniques described in Section 4. Typical values are in the range $5-20 \times 10^{40} \mathrm{Mx}^{2} / \mathrm{h}$ for ARs with a magnetic flux per polarity $\Phi \approx 10^{22} \mathrm{Mx}$ (Figs. 4, 7, 9, Chae et al., 2001; Nindos and Zhang, 2002; Nindos et al., 2003; Moon et al., 2002b; Chae et al., 2004; Hartkorn and Wang, 2004). The maximum computed rate value is $\approx 50 \times 10^{40} \mathrm{Mx}^{2} / \mathrm{h}$ (Nindos et al., 2003), still well below the first order of magnitude estimate of Wang. As expected, a lower rate is found in smaller ARs: in the range $0.1-1 \times 10^{40} \mathrm{Mx}^{2} / \mathrm{h}$ for ARs with $\Phi \approx 0.2-0.4 \times 10^{22} \mathrm{Mx}$ (Chae, 2001; Yamamoto et al., 2005). An exception is the result of Romano et al. (2005) who obtained a low mean rate $\approx 0.03 \times 10^{40} \mathrm{Mx}^{2} / \mathrm{h}$ while the AR studied has $\Phi \approx \times 10^{22} \mathrm{Mx}$.

Short term studies (less than one day) report variations which can be difficult 
to interpret (see Section 5.1). The studies based on several days (up to 6 days so that the AR stays close enough to disk centre) show various trends depending on the ARs. The clearest cases are when a rotating sunspot is present since there is a significant input of helicity that dominates other inputs. When the emergence of the AR is monitored from the beginning, a peak in the helicity rate is usually found such as in Fig. 4 (Nindos and Zhang, 2002; Chae et al., 2004; Yamamoto et al., 2005). This rate evolution is qualitatively similar to the one found in the MHD simulations of photospheric emergence of twisted flux tubes (Magara and Longcope, 2003; Gibson et al., 2004; Cheung et al., 2005). It would be certainly worth to compare observations and simulations more closely, in particular to gain insight into the twist of subphotospheric flux tubes. Finally, there are puzzling cases where the helicity rate reverses sign (Fig. 9, Chae, 2001; Chae et al., 2001; Nindos et al., 2003; Yamamoto et al., 2005). Is it a real reversal (not an artifact of the derived velocities)? Or is it due to emergence of a flux tube with the opposite magnetic helicity, or to photospheric vortex flows?

Active regions cover only a minor fraction of the solar surface. Quiet Sun areas have low magnetic flux density, but they still represent an important amount of the total absolute magnetic flux (comparable to ARs), but with many mixed polarities. Using a tracking algorithm applied to high cadence ( 5 minutes), high resolution SOHO/MDI magnetograms, Welsch and Longcope (2003) obtained a mean helicity flux density (per unit surface) of $\approx 2 \times 10^{16}$ $\mathrm{Mx}^{2} \mathrm{~cm}^{-2} / \mathrm{h}$ in five portions of the quiet Sun in years 1996-1997. This corresponds to an helicity rate of $\approx 0.05 \times 10^{40} \mathrm{Mx}^{2} / \mathrm{h}$ for a full solar hemisphere, which is typically two orders of magnitude below the rates found in ARs. This shows that the contribution of the quiet Sun to helicity injection by footpoint motions is negligible (this does not include the remnant of ARs after several months: see Section 5.3).

\subsection{Flux density}

It appears natural to define an helicity flux density (flux per unit surface), $G_{A}(\mathbf{x})$ at position $\mathbf{x}$, as the integrand of Eq. (27):

$$
G_{A}(\mathbf{x})=-2\left(\mathbf{A}_{p} \cdot \mathbf{u}\right) B_{n} .
$$

$G_{A}$ has been used in several studies to determine the spatial injection patterns of magnetic helicity in active regions (Chae, 2001; Chae et al., 2001; Kusano et al., 2002; Nindos and Zhang, 2002; Moon et al., 2002a,b; Nindos et al., 2003; Chae et al., 2004; Kusano et al., 2004a; Yamamoto et al., 2005). In all these different works, $G_{A}$ maps always appear extremely complex both in space and time, with $G_{A}$ polarities of both signs present at any time (Figs. 4,6). However, 


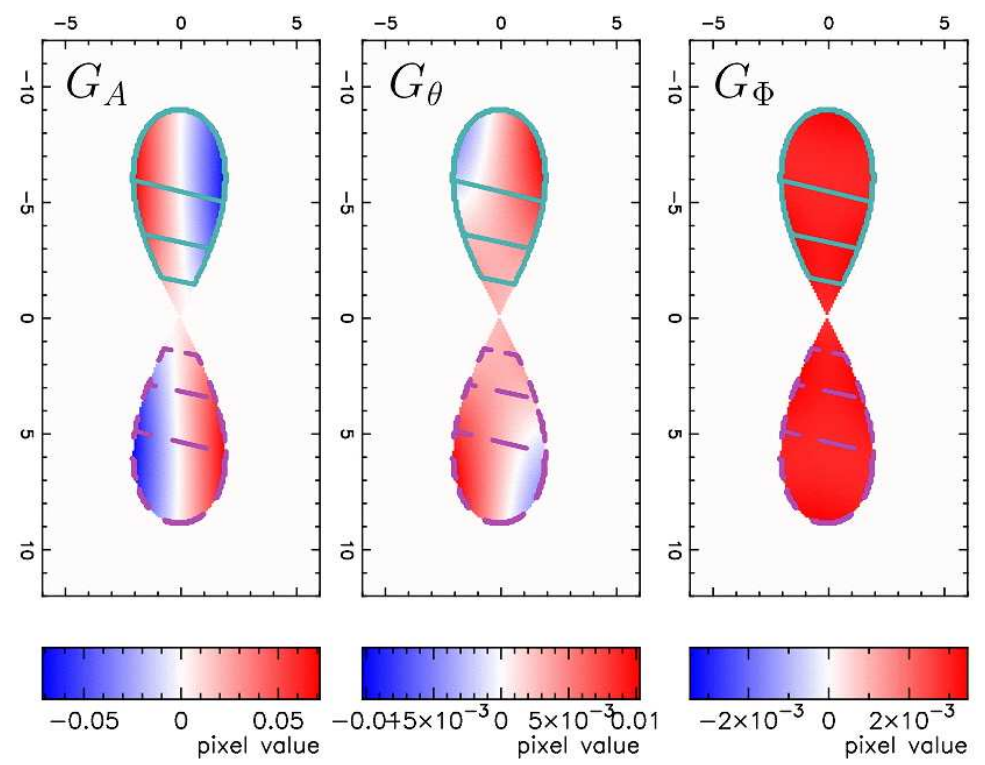

Fig. 5. Helicity flux density at the boundary $\mathcal{S}_{p}$ in the emergence of a twisted flux tube modeled by a half torus with a twist of 0.1 turns. The maps are shown when the bottom central part of the flux tube just crosses $\mathcal{S}_{p}$. Fake (unphysical) polarities of both signs fully dominate the $G_{A}$ map (notice the large positive/negative range of values compared to other maps), while they are much weaker with $G_{\theta}$ and they disappear with $G_{\Phi}$ (from Pariat et al., 2005).

$G_{A}$ is not a real helicity flux density since it introduces artificial polarities of both signs (Pariat et al., 2005).

It is possible to rewrite the helicity rate (Eq. 27) as a double integral over the surface which involves the relative rotation rate, $\mathrm{d} \theta\left(\mathbf{x}-\mathbf{x}^{\prime}\right) / \mathrm{d} t$, of pairs of elementary fluxes, $B_{n} \mathrm{~d} \mathcal{S}$ and $B_{n}^{\prime} \mathrm{d} \mathcal{S}^{\prime}$, located at the photospheric positions $\mathbf{x}$ and $\mathbf{x}^{\prime}$, respectively. For a planar surface one obtains:

$$
\left.\frac{\mathrm{d} H}{\mathrm{~d} t}\right|_{\mathcal{S}_{p}}=-\frac{1}{2 \pi} \int_{\mathcal{S}_{p}} \int_{\mathcal{S}_{p}} \frac{\mathrm{d} \theta\left(\mathbf{x}-\mathbf{x}^{\prime}\right)}{\mathrm{d} t} B_{n}(\mathbf{x}) B_{n}^{\prime}\left(\mathbf{x}^{\prime}\right) \mathrm{d} \mathcal{S} \mathrm{d} \mathcal{S}^{\prime} .
$$

Then, a new estimate of the helicity flux density is $G_{\theta}$ defined by:

$$
G_{\theta}(\mathbf{x})=-\frac{B_{n}(\mathbf{x})}{2 \pi} \int_{\mathcal{S}_{p}} \frac{\mathrm{d} \theta\left(\mathbf{x}-\mathbf{x}^{\prime}\right)}{\mathrm{d} t} B_{n}^{\prime}\left(\mathbf{x}^{\prime}\right) \mathrm{d} \mathcal{S}^{\prime} .
$$

Pariat et al. (2005) compared the properties of $G_{A}$ and $G_{\theta}$, applying them to several theoretical models: translational motions of a single polarity, separation and rotation of two polarities, and emergence of twisted flux tubes (Fig. 5). In all cases, they showed that $G_{A}$ almost always produces spurious signals that confuse the interpretation of the injection of helicity. Although $G_{\theta}$ produces 

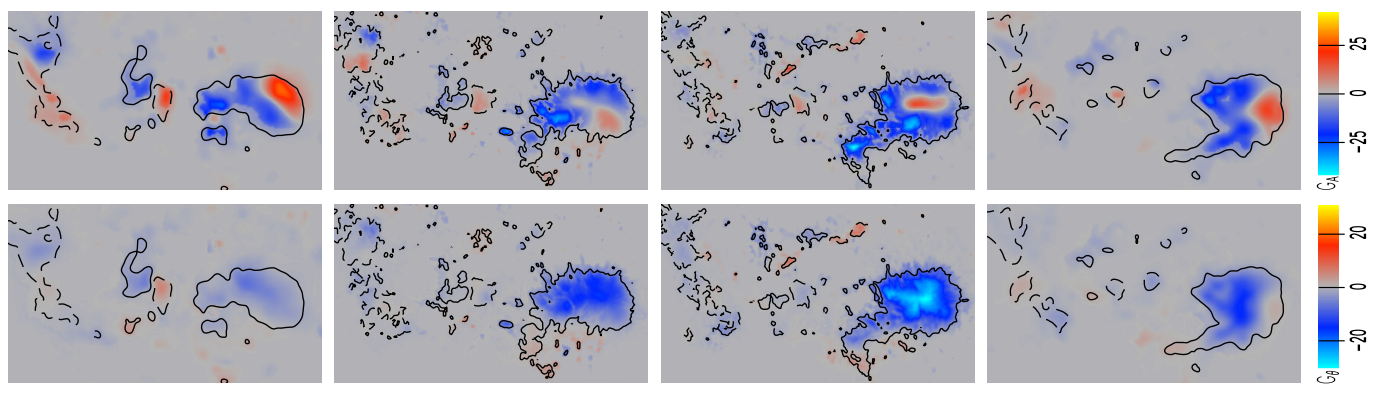

Fig. 6. Temporal evolution of $G_{A}$ (top row) and $G_{\theta}$ (bottom row) in AR 9114. $G_{A}$ has evolving polarities of both signs, while $G_{\theta}$ is mostly negative. The $G_{\theta}$ maps show that the absolute helicity rate is first increasing then decreasing in magnitude (in agreement with the numerical summation of the $G_{A}$ maps). The flux density maps are in units of $10^{6} \mathrm{~Wb}^{2} \cdot \mathrm{m}^{-2} \cdot \mathrm{s}^{-1}$ and have $\pm 300 \mathrm{G}$ isocontours of $B_{n}$ (from Pariat et al., 2006).

also some fake signals, they are usually weaker by more than one order of magnitude than those obtained with $G_{A}$. Pariat et al. (2006) applied these results to the ARs analyzed by Nindos et al. (2003), and they found that $G_{\theta}$ maps are almost unipolar, in sharp contrast of those of $G_{A}$ (Fig. 6).

While $G_{\theta}$ is of great practical use, it is still not the flux density of magnetic helicity. Indeed to define it one needs the coronal connectivities for all the elementary flux tubes. Then, the helicity flux per elementary flux tube can be defined. It can be rewritten as a flux of magnetic helicity per unit surface, called $G_{\Phi}$ (Pariat et al., 2005). $G_{\Phi}$ is a field-weighted average of $G_{\theta}$ at both photospheric footpoints, located at $\mathbf{x}_{ \pm}$, of the coronal connection:

$$
G_{\Phi}\left(\mathbf{x}_{ \pm}\right)=\frac{1}{2}\left(G_{\theta}\left(\mathbf{x}_{ \pm}\right)+G_{\theta}\left(\mathbf{x}_{\mp}\right)\left|\frac{B_{n}\left(\mathbf{x}_{ \pm}\right)}{B_{n}\left(\mathbf{x}_{\mp}\right)}\right|\right) .
$$

$G_{\Phi}$ was used to remove the remnant fake polarities present with $G_{\theta}$ in the case of an emerging flux nearby to another magnetic polarity (Pariat et al., 2006).

Just as $G_{A}$, both $G_{\theta}$ and $G_{\Phi}$ can be computed with any method used to estimate the photospheric velocities (Section 4). When local correlation tracking and/or the induction equation are used, one can deduced only the full helicity flux density, but if plasma motions are available the flux density can be derived separately for the tangential and normal terms (Eqs. 24,25). 


\section{Techniques to measure photospheric flows and helicity fluxes}

The determination of the helicity flux density requires the measurement of the plasma velocity $\mathbf{v}$ or of the flux-tube velocity $\mathbf{u}$ (Eq. 26) at the photospheric level. Doppler data provide only one component of $\mathbf{v}$ which is not enough to compute the helicity flux density. Other methods are required.

\subsection{Standard tracking methods}

At the photospheric level, the magnetic field is dominantly clustered in flux tubes (pores, sunspots), separated by low field regions. This defines magnetic features with different thermodynamic conditions, and so, brightness. In the feature tracking method, developed by Strous et al. (1996), these features are identified by local intensity extrema and followed in the next images (with the secure constrain that the motions do not exceed one pixel in between two successive images). The motions of the flux tubes are then derived if the brightness evolution follows well the magnetic evolution. This method is well adapted for high resolution observations of emerging fluxes (since they are usually split in thin flux tubes). Compared to the methods solving either the advection or the induction equations, the problem is not undetermined because the individual structures are supposed to be always traceable so that an extra information is implicitly added to the data, the labeling of the magnetic structures. This works very well if the magnetic structures are isolated and evolve independently (e.g. no merging, no splitting), at the expense of not determining the internal velocity in the structures.

A more widely used method is the local correlation tracking (LCT). Originally, LCT was developed for intensity images (November and Simon, 1988) and Chae (2001) first applied it to magnetograms. The method can be shortly summarized as follows. A local sub-image is first defined with a localized apodisation function peaked at position $\mathbf{x}$ in the magnetogram taken at time $t$. The apodisation function decreases toward the edges of the sub-image and it is mostly characterized by its full width at half maximum (FWHM). This width defines the effective window. This sub-image is cross correlated with another sub-image centered at $\mathbf{x}+\Delta \mathbf{x}$ in the next magnetogram (taken at $t+\Delta t$ ). The best correlation found, over the sub-images of the second magnetogram in the vicinity of $\mathbf{x}$, defines the LCT velocity $\Delta \mathrm{x} / \Delta t$. A velocity map is build by repeating the procedure for all $\mathbf{x}$ positions.

The LCT assumes that the photospheric magnetic field is advected by large scale flows, so this method gives an approximate solution to the advection 
equation:

$$
\frac{\partial B_{n}}{\partial t}+\mathbf{u} \cdot \nabla B_{n}=0
$$

with $\mathbf{u} \approx \Delta \mathbf{x} / \Delta t$. Since the sub-image is globally translated, only an average of the velocity is obtained at the spatial resolution $(\approx$ FWHM). Moreover, the cross-correlation needs spatial fine structures in the sub-images to be efficient, so the LCT implicitly assumes the hierarchy:

$$
l_{B}<<\mathrm{FWHM}<<l_{u}
$$

where $l_{B}$ and $l_{u}$ are the typical length scales of the magnetic and velocity fields. In applications this hierarchy is only partially satisfied and the derived velocities are affected by the choice of the FWHM.

The time step $\Delta t$ should be selected carefully. If $\Delta t$ is too small, the magnetic evolution is insignificant and the determined velocities are dominated by noise. If $\Delta t$ is too large, the transformation of the magnetograms is too important to give good correlations, it is then hazardous to follow the flux tubes. This is particularly important in emerging regions around the magnetic inversion line: the apparent motion $\mathbf{u} \approx-v_{n} \mathbf{B}_{t} / B_{n}$ can be large. In the emerging region, the optimal $\Delta t$ is much lower than in the other parts of the magnetogram. A compromise needs to be found, which implicitly filters the derived velocities.

The large number of computed cross-correlations, needed for a time series of magnetograms, implies that the LCT algorithm is slow. The computation speed has been improved by Welsch et al. (2004) with a fast Fourier correlation, and by Potts et al. (2004) with a ball tracking technique.

For a given $B_{n}$ time series, the advection Eq. (32) is a scalar equation for the two unknown components of $\mathbf{u}$. How LCT solves this under-determined system? It is through a local model of the velocity field within the apodising window. The usual one is a uniform velocity (Schuck, 2005). The $N$ pixels within the apodising window are supposed to satisfy Eq. (32) with the same $\mathbf{u}$ velocity. This strong hypothesis transforms the problem to an over-determined system ( $N$ equations, two unknowns) and the LCT satisfies the advection equation only in a least-square sense. This is explicitly implemented in the socalled differential LCT. It uses finite differences of the data (as all techniques described in the two next sections). While differentiation of data is a step which enhances the noise over the signal, the differential LCT performed better than the LCT even in very noisy data because it also performs a least-square fit within the apodising window (Schuck, 2006). The constraint of uniform velocity can be relaxed to include local linear spatial dependencies (called an 
affine flow). This weakens the effect of the apodising window on the determined velocities.

\subsection{Tracking using the induction equation}

The measurement of the photospheric flows is improved by introducing the physics of the magnetic evolution as given by the induction equation:

$$
\frac{\partial \mathbf{B}}{\partial t}=\nabla \times(\mathbf{v} \times \mathbf{B})+\eta \nabla^{2} \mathbf{B}
$$

Even with a uniform resistivity, the resistive term cannot be determined with present observations, and the estimation of the velocity relies on the ideal induction equation. Moreover, this equation can tell nothing about the component of $\mathbf{v}$ parallel to $\mathbf{B}$. This component has also no effect on the helicity flux density, so in fact we have only two independent unknowns. Since the gradient of an arbitrary function can be added to $\mathbf{v} \times \mathbf{B}$, without affecting $\partial \mathbf{B} / \partial t$, finding $\mathbf{v}$ from Eq. (34) with $\eta=0$ and from a temporal series of magnetograms is an under-determined problem. Moreover, vertical gradients are difficult to estimate (they depend on atmospheric models) and in practice we can rely only on the vertical component of the ideal induction equation, which has two equivalent forms:

$$
\begin{aligned}
& \frac{\partial B_{n}}{\partial t}=\nabla_{t} \cdot\left(v_{n} \mathbf{B}_{t}-B_{n} \mathbf{v}_{t}\right), \\
& \frac{\partial B_{n}}{\partial t}=-\nabla_{t} \cdot\left(B_{n} \mathbf{u}\right),
\end{aligned}
$$

The last form outlines most clearly that we have two unknowns (the two components of $\mathbf{u}$, since $\mathbf{u}_{n}=0$, by definition) for only one scalar equation (analogue to the under determination of the advection equation). The LCT solves a transport equation (Eq. 32) which is not compatible with the physical evolution of the photospheric field (Eq. 36).

Schuck (2005) developed a method to solve the induction equation in the spirit of differential LCT. Here a local affine velocity model is needed (allowing linear spatial deformations of the velocity within the apodising window). The determined $\mathbf{u}$ satisfies Eq. (36) in a least square sense within the apodising window. This method is called the differential affine velocity estimator (DAVE). It incorporates the advantages of differential LCT, including a quantitative measure of the goodness of the affine flow model. Schuck (2006) demonstrated that it outperforms the classical LCT approach on the synthetic and noisy test data considered. With its ability to detect flow patterns inside the apodising 
window, its compatibility with the induction equation and its allowance of high time cadence images (this is permitted since it is robust against noise), DAVE is a promising technique. A first application to an already well studied AR gives detailed flow patterns which could not be detected by previous methods, in particular the vortex flow in a rotating spot is intermittently detected.

\subsection{Global solution of the magnetic induction equation}

Other methods solve Eq. (35) or (36) at each magnetogram pixel. They all introduce an extra information to solve the undetermined problem.

Kusano et al. (2002) proposed the first method, called IE, to solve Eq. (35). It is extended and clarified by Kusano et al. (2004a). Below, for comparison with other methods and for conciseness, I rather present the IE method transformed to solve Eq. (36). The method can be extended to a large set of two dimensional fields $\hat{b}$ defined with the constraint $|\hat{b}|=1$ on $\mathcal{S}_{p}$. Decomposing $\mathbf{u}$ on $\hat{b}$ and $\hat{n} \times \hat{b}$, Eq. (36) becomes:

$$
\frac{\partial B_{n}}{\partial t}=\nabla_{t} \cdot\left(B_{n} u_{\|} \hat{b}+B_{n} u_{\perp} \hat{n} \times \hat{b}\right)
$$

This equation can have a unique solution for $B_{n} u_{\|}$if $B_{n} u_{\perp}$ is provided independently (e.g. by magnetograms and LCT) as follows. Let us suppose that $B_{n} u_{\|}$and $B_{n} u_{\|}^{\prime}$ are two solutions, then

$$
B_{n}\left(u_{\|}-u_{\|}^{\prime}\right) \hat{b}=\nabla_{t} \times(\xi \hat{n})
$$

since the two other term in Eq. (37) are supposed to be known. Applying successively $\hat{n} \times$ and $\hat{b}$. to Eq. (38), gives:

$$
\hat{b} \cdot \nabla_{t} \xi=B_{n}\left(u_{\|}-u_{\|}^{\prime}\right) \hat{b} \cdot(\hat{n} \times \hat{b})=0 .
$$

So $\xi$ is constant along field lines of $\hat{b}$ on $\mathcal{S}_{p}$. If $\hat{b}$ has a null point where field lines meet, $\xi$ is constant in the domain linked by $\hat{b}$ field lines to the null point. In this domain $\nabla_{t} \times(\xi \hat{n})=0$ and Eq. (38) gives $u_{\|}=u_{\|}^{\prime}$ demonstrating the uniqueness of the solution (see Section 3.2 of Kusano et al. (2002) for further discussions on the uniqueness).

The selection of the $\hat{b}$ field provides a freedom to select the contribution of the LCT $\left(u_{\perp}\right)$. Since the large horizontal motions present near the inversion line $\left(B_{n}=0\right)$ are difficult to measure by LCT, it is better to select $\hat{b}$ so that the emerging motions are provided only by the induction equation. This choice is 
$\hat{b}=\mathbf{B}_{t} / B_{t}$ since for pure emergence $\mathbf{u}=-v_{n} \mathbf{B}_{t} / B_{n}$ so that $u_{\perp}=0$. Moreover $\mathbf{B}_{t}$ has usually star null points on each magnetic concentration, and the nulls are linked by field lines of $\mathbf{B}_{t}$ insuring uniqueness in the full domain. In the emerging case, the IE method is far superior to the LCT method to recover the correct helicity flux density (Kusano et al., 2004a). The above formulation has the advantage to involve only the direction of the transverse field $\mathbf{B}_{t}$, the most accurate measured part of $\mathbf{B}_{t}$, rather than $\mathbf{B}_{t}$ itself as in Kusano et al. (2004a). This implies solving the induction equation for $B_{n} u_{\|}$(rather than for $\left.u_{\|}\right)$to avoid the singularity of $u_{\|}$at inversion lines $\left(B_{n}=0\right)$.

Welsch et al. (2004) proposed another method, called ILCT. It is based on the Helmholtz's decomposition of the two-dimensional vector $B_{n} \mathbf{u}$ as (Amari et al., 2003a):

$$
B_{n} \mathbf{u}=-\nabla_{t} \phi+\nabla_{t} \psi \times \hat{n} .
$$

The last term gives the rotational contribution of $B_{n} \mathbf{u}$ which is most effective for injecting magnetic helicity. $\phi$ is provided by the induction equation: including Eq. (40) in Eq. (36), one has:

$$
\triangle_{t} \phi=\frac{\partial B_{n}}{\partial t}
$$

where $\triangle_{t}$ is the perpendicular Laplacian. $\psi$ is given by the LCT method: taking $\nabla_{t} \times$ of Eq. (40) and setting $\mathbf{u}=\mathbf{u}_{\mathrm{LCT}}$, one gets:

$$
\triangle_{t} \psi=-\nabla_{t} \times\left(B_{n} \mathbf{u}_{\mathrm{LCT}}\right)
$$

The advantage of the ILCT method is that Eqs. $(41,42)$ can be solved in Cartesian geometry by the standard Fourier transform, which are fast, while the IE method of Kusano et al. needs an iteration procedure. However, the advantage of the IE method is to minimize the input of the LCT, in particular computing all the emerging flows with the induction equation. With the decomposition of Eq. (40), the helicity rate (Eq. 27) simplifies to (Amari et al., 2003a):

$$
\left.\frac{\mathrm{d} H}{\mathrm{~d} t}\right|_{\mathcal{S}_{p}}=-2 \int_{\mathcal{S}_{p}} \psi B_{n} \mathrm{~d} \mathcal{S},
$$

so the ILCT gives an helicity rate coming only from the LCT, in sharp contrast with the IE method which favors the contribution coming from the induction equation. 
Longcope (2004) decomposed the velocity as in Eq. (40), solved $\phi$ from Eq. (41), but computed $\psi$ by minimizing the kinetic energy of $\mathbf{v}-\mathbf{w}$, where $\mathbf{w}$ contains the velocity components measured by other means (e.g. LCT, Doppler shifts). When a component is not measured, it is set to zero. More precisely, Longcope minimizes the functional

$$
W=\frac{1}{2} \int_{\mathcal{S}_{p}}\left(\left(\mathbf{v}_{t}-\mathbf{w}_{t}\right)^{2}+\left(v_{n}-w_{n}\right)^{2}\right) \mathrm{d} \mathcal{S},
$$

under the variation of $\psi$ and $v_{n}, \mathbf{v}_{t}$ being replaced by $\left(v_{n} \mathbf{B}_{t}-\nabla_{t} \phi+\nabla_{t} \psi \times\right.$ $\hat{n}) / B_{n}$. This method has a computed velocity $\mathbf{v}$ compatible with the induction equation while including partial velocity informations from other sources. With the input of Doppler velocities in $\mathbf{w}$, this technique can determine the plasma velocity, and separate the two helicity flux contributions of Eqs. (24, $25)$. However, since the method minimizes $W$ to find $\psi$, it is expected to give only an under-estimation of the helicity rate (Eq. 43).

Georgoulis and LaBonte (2006) introduced another constraint to removed the ambiguity of the induction Eq. (35). They assumed that, at each point of the vector-magnetogram, the cross-field plasma velocity $\mathbf{v}_{\perp}$ is in the plane defined by $\mathbf{B}$ and the cross field gradient $(\nabla B)_{\perp}$ :

$$
(\nabla B \times \mathbf{B}) \cdot \mathbf{v}=0
$$

They called the method "minimum structure reconstruction" (MSR). It can include Doppler data to determine the field-aligned flows (but they are not needed to compute the helicity flux density). The justifications of Eq. (45) and its inclusion in solving the induction equation are too complex to be reported here. They argued that Eq. (45) implies that the vertical component of $\mathbf{v}_{\perp}$ vanishes. This limits the application of the method to cases where the helicity flux density is dominantly coming from horizontal plasma motions (and not from emergence).

\subsection{Present limitations}

While the above methods have lead to very important advances, and are presently the most accurate way to track the evolution of the coronal magnetic helicity, it is also important to keep in mind the present limitations. On top of the uncertainties coming from the magnetic field measurements, some others are linked to:

- the spatial resolution, 
- the regions with absence of significant contrast,

- undetectable motions.

The spatial resolution is certainly the main concern since, to compute the helicity rate, we need to follow the evolution of magnetic flux tubes, which can be as thin as $100 \mathrm{~km}$ outside sunspots, while the actual pixel size of magnetograms is typically 10 times larger. When an ensemble of unresolved flux tubes are observed as a nearly continuous pattern, one may wonder about the significance of the deduced velocities (they will include, at most, only the global motion of the flux tubes). This resolution problem is amplified with the LCT where the FWHM of the sub-images is typically 4 magnetogram pixels.

The second point may also have a considerable impact on the magnitude of the computed rates since it concerns the sunspots, where a large fraction of the magnetic flux of active regions is present (before their decay). This is amplified by the usual non-linear response (saturation-like) of magnetographs at high field values. Test configurations, without internal structure, can be easily constructed, e.g. simulating the emergence of a twisted flux tube. Gibson et al. (2004) found that the LCT technique yields an underestimation of the helicity by an order of magnitude by the end of the flux tube emergence.

The third point is linked to the part of the plasma motions which are not changing the $B_{n}$ distribution, so this part will not be detectable by all the present methods (except with Doppler velocities). This corresponds to the part of $v_{n} \mathbf{B}_{t}-B_{n} \mathbf{v}_{t}=-B_{n} \mathbf{u}$ in Eqs. $(35,36)$ that can be written as $\nabla f \times \hat{n}$, where $f$ is an arbitrary function. A particular case is rotational motions along isocontours of $B_{n}$ and such motions are very efficient to transfer magnetic helicity!

Finally, at first sight, Doppler velocities seem to be an important input to helicity rate computations, in particular in emerging regions. However, the input of Doppler velocities requires an estimation of the plasma velocity $\mathbf{v}$ (3 components) rather than the footpoint motion $\mathbf{u}$ ( 2 components). Then, if we use also the Doppler velocity data, we still have the same level of underdetermination (one velocity component) for the induction equation given by the equivalent Eqs. $(35,36)$. It should also be kept in mind that most of the Doppler velocity is coming from plasma moving along $\mathbf{B}$, in particular draining down along the emerging flux tubes. These parallel flows do not contribute to the helicity flux density and a very accurate determination of the magnetic field orientation is needed to deduce the velocity component orthogonal to $\mathbf{B}$. 

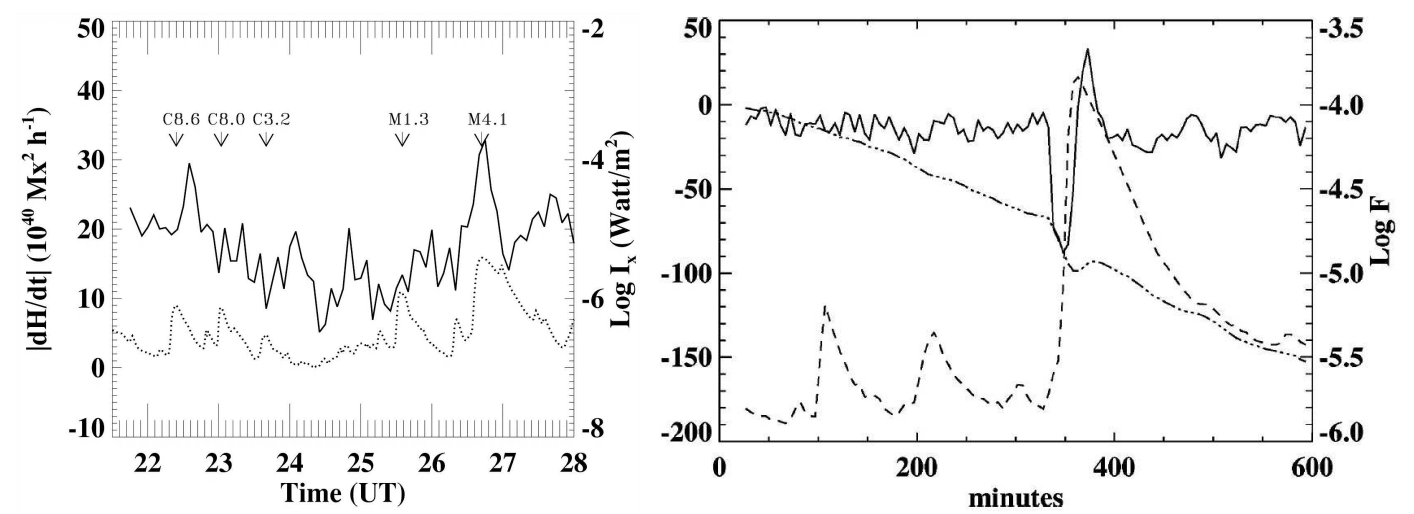

Fig. 7. Photospheric rate of magnetic helicity in flaring ARs (continuous line) compared to the GOES X-ray flux (dotted or dashed line). The left panel is from Moon et al. (2002a). The right panel is from Hartkorn and Wang (2004) and has a third curve (dashed-dotted line) representing the accumulated helicity. The impulsive rate during the flare modifies only weakly the general evolution (also true for other flares). The impulsive variation during flares of the helicity rate is most probably a fake signal due to the perturbations of the magnetograph spectral line.

\section{Implications for solar phenomena}

Together with the developments of practical methods to compute magnetic helicity and its photospheric flux, the first applications to various events have been achieved (the earlier applications are better explained in the review of van-Driel Gesztelyi et al., 2003).

\subsection{Flares}

Nindos and Andrews (2004) extrapolated with a linear force-free field the photospheric field of a large set of flaring ARs, and found the value of $\alpha$ which best fitted each coronal loop. They found that confined flares (without an associated CME) are more often present in ARs which have mixed sign of $\alpha$. This could indicate the presence of magnetic helicity with mixed sign. This is coherent with the theoretical expectation that cancellation of magnetic helicity leads to more free magnetic energy available for flaring (e.g. Kusano et al., 2004b). However, a large range of $\alpha$, in particular with opposite sign values, means that the field is far from a linear force-free field. Moreover, a mixed sign of $\alpha$ does not necessarily imply a mixed sign of $H$. Then, this result needs to be tested independently, for example, with maps of the photospheric flux density of helicity using $G_{\theta}$, and if possible $G_{\Phi}$, to avoid the fake mixed polarities of $G_{A}$ (Section 3.3). 
Moon et al. (2002a) computed the photospheric helicity rate with velocities derived from the LCT method (Section 4.1). In one AR, they showed that the logarithm of the X-ray flux emitted during each flare is almost linearly correlated with the accumulation of magnetic helicity injected since the time of previous flare. In the next publication, Moon et al. (2002b) found a similar relation in another AR, but for the impulsive helicity rate injected during the flare itself. The physical interpretation is different in both cases and further studies are needed to clarify this.

Several studies have pointed out an impulsive photospheric helicity rate during flares (Moon et al., 2002a,b; Nindos et al., 2003; Hartkorn and Wang, 2004; Romano et al., 2005). Contrasting results are present, for example, Moon et al. found it almost systematically, even for M-class flares, while Hartkorn and Wang found it only in two of three X-flares and never in M-flares. What is striking is the time correlation between the helicity rate and the X-ray flux (Fig. 7). Moon et al. argued for an extra input of helicity during flares, while Hartkorn and Wang concluded, in agreement with the results of Qiu and Gary (2003), that this is an artificial signal due the impact of particle beams on the atmosphere (this modifies the spectral line used by the magnetograph). It is also worth noting that the helicity peak has a width at half maximum comparable to the LCT time cadence, 20 minutes, so that the effect is artificially broaden in time. Moreover the origin of the helicity peak is very localized in the AR where the flare is located. A detailed comparison of the helicity flux density maps with the observed flare ribbons and flare loops is needed to fully clarify the origin of the reported impulsive variations.

\subsection{CMEs}

CMEs are thought to be the last physical mechanism in the chain of processes which transfer magnetic flux and helicity from the base of the convective zone (from the dynamo region) into the interplanetary medium. Both magnetic flux and helicity are injected from the convective zone into the corona in a systematic, though bursty, way. Magnetic helicity is expected to accumulate with time in the corona for the following points. First, magnetic helicity is injected with a dominant sign in each hemisphere $(<0$ in the north, $>0$ in the south, see Section 2.3). Second, magnetic bipoles reverse their sign with the solar cycle while magnetic helicity keeps the same dominant sign in each hemisphere. These two results imply an average helicity input with a fixed sign in each hemisphere. Third, the events, where opposite magnetic helicities can be canceled, involve only a small fraction of the magnetic flux (e.g. between ARs of opposite helicity sign, either within the same hemisphere or across the equator, Pevtsov, 2000). Four, also a small amount of magnetic flux is involved in reconnection between ARs and coronal holes, so plausibly a low fraction of 
magnetic helicity is lost from the corona by this mechanism (but this has to be quantified). Finally, magnetic helicity is well preserved even when magnetic energy is dissipated (Section 3.1). With all these constraints, Rust (1994) and Low (1997) advocated that the Sun can only avoid endless accumulation of helicity in the solar corona by ejecting the accumulated helicity via the launch of CMEs. An even stronger conjecture is that a CME is launched when a threshold of magnetic helicity is reached.

Zhang et al. (2006) analyzed the maximum amount of magnetic helicity that can be stored in an axisymmetric force-free field outside a sphere. The $B_{n}$ distribution on the sphere is a given dipolar field, and they studied various shear profiles, increasing the azimuthal field. The series of equilibrium saturate at nearly the same amount of magnetic helicity for different shear profiles, while the magnetic energy has substantial differences. Using an extension of Cauchy-Schwartz inequality, they showed that the coronal magnetic helicity is bounded by $2 \Phi \Phi_{\varphi}$, where $\Phi$ is the positive magnetic flux crossing the photosphere (half an hemisphere) and $\Phi_{\varphi}$ is the total azimuthal magnetic flux in the corona. Using the maximum $\Phi_{\varphi}$ found numerically, this means $H / \Phi^{2} \lesssim 0.54$ (notice that this is a global value, so a kind of "average" between the twisted core and the more potential surroundings). An even lower maximum value is found when the maximum $H$ found numerically is used: $H / \Phi^{2} \lesssim 0.35$. This upper bound is indeed just above the maximum found in ARs, $H / \Phi^{2} \lesssim 0.2$ (see Section 2.5) ! Moreover, Jacobs et al. (2006) performed MHD simulations by shearing an axisymmetric arcade outside a sphere. They considered the large scale dipole of the Sun and they tested several models of the solar wind. Their results are slightly dependent on the wind model used. They found the formation, then ejection, of a twisted flux tube when $H / \Phi^{2}$ is typically above 0.2-0.3, implying that a relative low global magnetic helicity is necessary for the production of CMEs (the helicity threshold can be slightly lowered by the effect of the solar wind on the large scale dipole).

Amari et al. (2003a) performed MHD simulations of a magnetic bipole in cartesian geometry. Magnetic helicity is first injected by twisting motions at the lower boundary. The stability of the configuration is tested by relaxations with no boundary motions. Finally, converging motions towards the magnetic inversion line are introduced. In this phase no magnetic helicity is injected, but magnetic reconnection is allowed, in particular at the boundary where cancellation of magnetic flux occurs. If the amount of helicity injected in the first phase is high enough, the magnetic configuration becomes unstable and the arcade is partially transformed in a twisted flux tube which is ejected. The same result is obtained with the last phase replaced by a diffusion of $B_{n}$, with the only main difference that the twisted flux tube can be in equilibrium for some time before its ejection (Amari et al., 2003b). The accumulation of helicity is a necessary but not sufficient condition for eruption in these evolutions where the unsigned magnetic flux crossing the boundary decreases. 

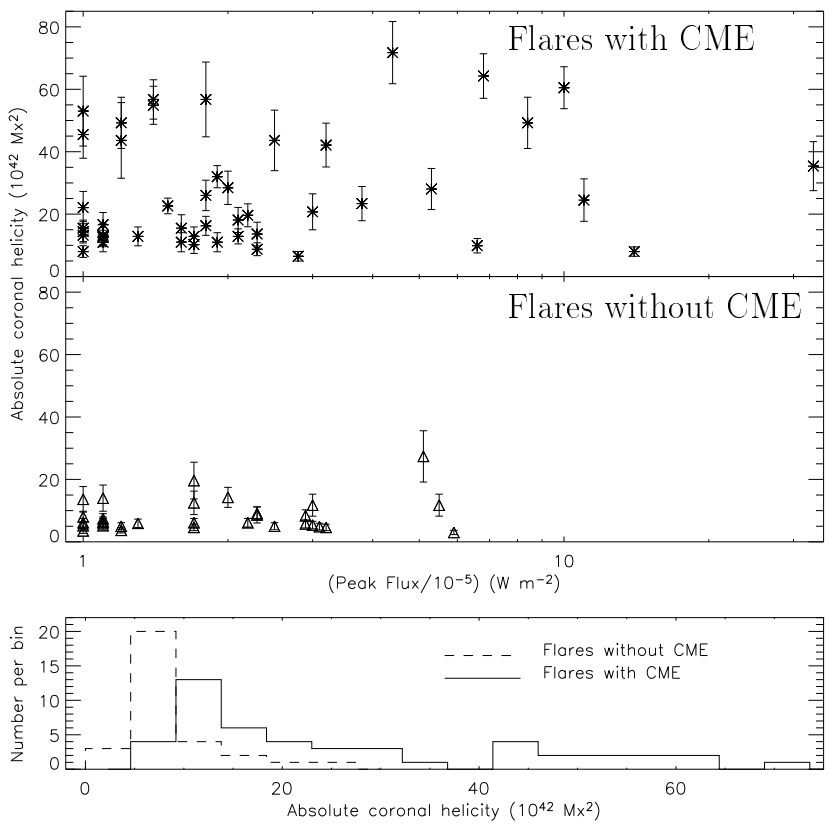

Fig. 8. Top panels. Preflare magnetic helicity stored in the AR coronal field (with a linear force-free field extrapolation) as function of the flare X-ray flux peak. The upper (lower) panel is for the flares with (without) associated CMEs. Bottom panel. Histogram of the coronal helicities splitting the flares with and without associated CMEs (from Nindos and Andrews, 2004).

Phillips et al. (2005) have repeated a breakout simulation (axisymmetric quadrupolar configuration outside a sphere) by designing the photospheric motions to inject no global magnetic helicity. They showed that the eruption occurs at nearly the same magnetic energy threshold as in a previous simulation where only positive helicity was injected. The amount of magnetic helicity has no role in their simulations because the negative and positive helicity regions are not reconnecting. While Kusano et al. (2004b) found contrasting results. They considered a sheared arcade with a reversed shear near the inversion line. The reconnection between these opposite helicity regions gives a nearly potential field that shrinks down quickly. This implies a local collapse of the large scale arcade, a second reconnection, and the creation of a twisted flux tube which is ejected. In this MHD simulation, the introduction of the reverse helicity is fundamental for the eruption to occur, at the opposite of the results of Phillips et al. (2005) for another configuration.

The previous theoretical results are controversial. What observations tell us? Nindos and Andrews (2004) considered all the ARs in the years 1996-1999 which produced $\mathrm{M}$ or X-class flares and which were observed sufficiently close to the central meridian $\left( \pm 50^{\circ}\right)$. They computed the coronal helicity with a linear force free field which best fit the coronal loops (Section 2.5). They found that the preflare helicity is significantly higher $\left(H=27 \pm 18 \times 10^{42} \mathrm{Mx}^{2}\right)$ in ARs having CMEs than in ARs having only confined flares $\left(H=8 \pm 5 \times 10^{42} \mathrm{Mx}^{2}\right)$; 
see Fig. 8. This is in agreement with the MHD simulation results of Amari et al. (2003b) where confined events occur only for low $\mathrm{H}$ values.

The above summary shows that the role of magnetic helicity in the launch of CMEs is still an open debate. While the accumulation of magnetic helicity of a given sign is a favorable condition to reach an instability, it is not a sufficient one. Other aspects are important, such as the location where this helicity is injected and where efficient reconnection is allowed to proceed (e.g. to decrease the stabilizing anchorage in the photosphere), so the magnetic topology of the configuration and the boundary motions are also key ingredients.

\subsection{Budget of helicity in ARs}

What is the amount of magnetic helicity involved in an AR during its full existence? This can be estimated by following the long-term evolution, from emergence to dispersion of isolated ARs and by recording their CME productivity. During the entire lifetime of two ARs (6 and 5 solar rotations, respectively) such counts have been done: NOAA 7978 was found to be the source region of 31 CMEs (Démoulin et al., 2002a), while NOAA 8100 produced 65 CMEs (Green et al., 2002). These numbers include corrections for data gaps and far-side locations of the ARs (using the mean CME rate computed from the AR disk transit). They are much higher than previously thought based on pre-SOHO observations (4-5/AR/lifetime), and imply a huge amount of (unsigned) helicity ejected from these ARs into the interplanetary space: $\approx 60-250 \times 10^{42} \mathrm{Mx}^{2}$ and $\approx 130-520 \times 10^{42} \mathrm{Mx}^{2}$ respectively, where an average MC helicity is used as an estimate of the average CME helicity $\left(H \approx 2-8 \times 10^{42} \mathrm{Mx}^{2}\right)$. This corresponds to $H / \Phi^{2}$ of about one turn. This amount of twist is coherent with the theoretical studies of Emonet and MorenoInsertis (1998) and Abbett et al. (2000) who showed that flux tubes need to be twisted enough to be able to survive their rise through the convection zone.

The above amount of coronal helicity in ARs is typically a factor 10 larger than the helicity provided by differential rotation shearing the AR configuration. This is confirmed by independent estimate of the coronal helicity using photospheric helicity rates (Chae et al., 2001; Nindos and Zhang, 2002; Nindos et al., 2003). Let us consider a simple magnetic bipole to illustrate why differential rotation is inefficient to provide a significant amount of helicity. Differential rotation both rotates magnetic polarities on themselves and their relative positions, introducing twist and writhe helicity fluxes, respectively. These fluxes always have opposite signs, while their magnitudes are similar, thus they partially cancel (Démoulin et al., 2002b). The relative importance of twist and writhe helicity injection by differential rotation depends mainly on the orientation of the bipole (with a change of dominance when the bipole 


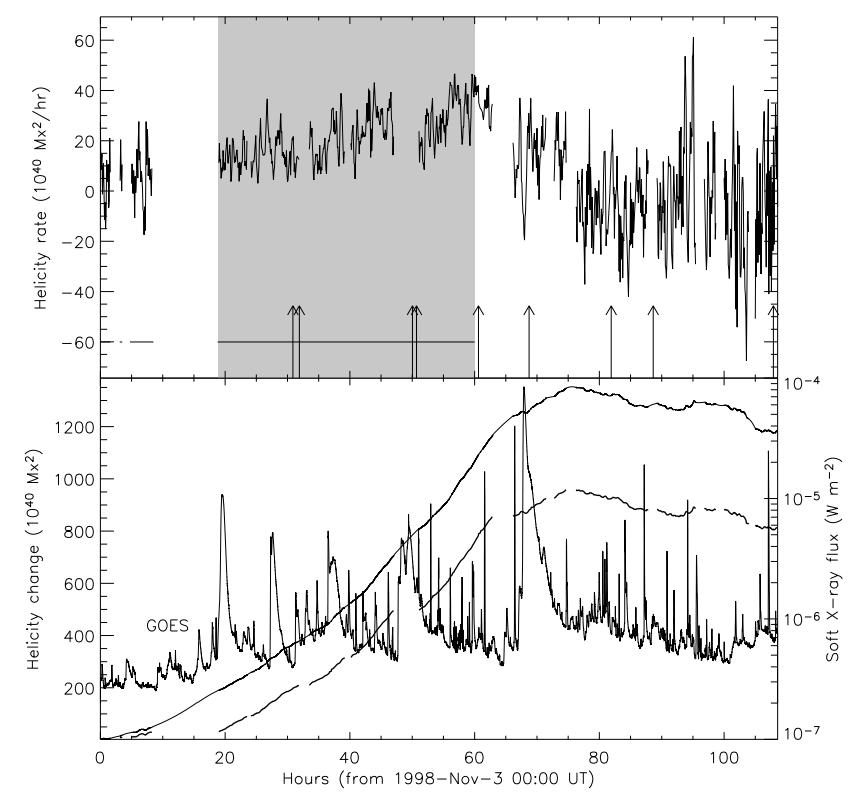

Fig. 9. Photospheric injection of magnetic helicity and CME production in a mature AR. Top panel. Helicity rate computed with LCT and MDI (in the gray area the high resolution data are used, resulting in less fluctuating results). The arrows indicate the first detection of the CMEs with LASCO). Bottom panel. Accumulated helicity calculated directly from the top panel (thick line) and from a spline interpolation to avoid data gaps (thin line). The evolution of X-ray flux observed by GOES is added with a logarithm scale (from Nindos et al., 2003).

tilt is $45^{\circ}$ on the equator).

The main source of the coronal magnetic helicity is thought to be the helicity contained in the subphotospheric flux tube forming the AR. As the flux tube is emerging in the corona, it also necessary bring part of its magnetic helicity. The helicity can also be transferred by torsional Alfvén waves when there is an unbalance of magnetic torque between the subphotospheric and the coronal parts of the flux tube (Longcope and Welsch, 2000; Chae et al., 2003). The expansion of the field in the corona decreases its torque, inducing a photospheric transfer and an Alfvén torsional wave propagating along the sub-photospheric part of the flux tube. It is present until the coronal field has a torque large enough to balance the subphotospheric torque. This model was tested by following the linear force-free $\alpha$ coefficient (determined by a best fit to coronal loops) in emerging ARs (Pevtsov et al., 2003). After typically one day, $\alpha$ usually saturates to a maximum while the magnetic flux growth continues. For the long-term evolution of ARs (time scale of months), such kind of helicity transfer is probably needed since the number of CMEs ejected is still significant, at the difference of strong flares the number of which decreases rapidly with the AR age (Démoulin et al., 2002a; Green et al., 2002). 


\subsection{Relation $C M E-M C$}

The above estimate of the helicity ejected by CMEs during the entire lifetime of two ARs are subjected to large uncertainties: the ones present for each MC (Section 2.6), but also the use of a mean helicity value for MCs not related to the AR studied. However, the distribution of MC helicities is very broad (Fig. 3). Then, it is important to analyze the MCs coming from the studied AR.

Nindos and Zhang (2002) first studied the magnetic helicity of MCs related to their solar source. They computed the photospheric helicity rate with LCT of an emerging AR during 4 days. During this period, the region produced $8 \mathrm{CMEs}$, and one was detected as a MC, a huge one, carrying about $H \approx$ $64 \times 10^{42} \mathrm{Mx}^{2}$ for a length of $1 \mathrm{AU}$. Either using this helicity or the average one of MCs detected in the year $2000\left(H \approx 8 \times 10^{42} \mathrm{Mx}^{2}\right)$, Nindos and Zhang (2002) found that the photospheric helicity rate is a factor 8-64 lower than helicity estimated in the 8 CMEs! Then, Nindos et al. (2003) extended this study by a detailed analysis of 6 ARs (one is presented in Fig. 9). A MC was observed from each of them. The helicity content of these MCs is broad: $H \approx 1-19 \times 10^{42} \mathrm{Mx}^{2}$ with a length estimated by the condition for the kink instability threshold (set to 2 turns). Using the MC helicity associated to each AR as an estimate for the CME helicity, Nindos et al. (2003) found a photospheric helicity injection broadly compatible with the ejected helicity in CMEs both with a length set to 0.5 AU or estimated from the kink threshold. These results show that a better agreement is found between the coronal and interplanetary estimate of helicity when the data from the AR where the MC originated are used.

The next step is to analyze the variation of the coronal helicity during a halo CME event together with the associated MC. So far this has been done only for two cases, a tiny and a large MC. Mandrini et al. (2005) analyzed the full evolution of a tiny AR well isolated from others and located close to the solar disk centre. The photospheric bipole emerged and dispersed at the photospheric level in less than 2 days. The variation of the coronal helicity during the eruptive event was estimated by using a linear force-free field fitted to the coronal loops. The link of the observed tiny $\mathrm{MC}$ to the AR was supported by the timing, the magnetic field orientation and the amount of magnetic flux involved (measured in the dimming regions and in the MC, Fig. 10). Moreover, the decrease of coronal helicity and the MC helicity were found in the same range: $\approx 2-3 \times 10^{39} \mathrm{Mx}^{2}$ (a tiny amount compare to the above values!). Dasso et al. (2006) re-analyzed in a similar way a well studied MC, observed on 18-20 October 1995. In this case the MC helicity, at least $10^{43} \mathrm{Mx}^{2}$, is larger by a factor $\approx 2$ than the variation of the coronal helicity in the associated CME event (Luoni et al., 2005). These studies show that the interplanetary and 


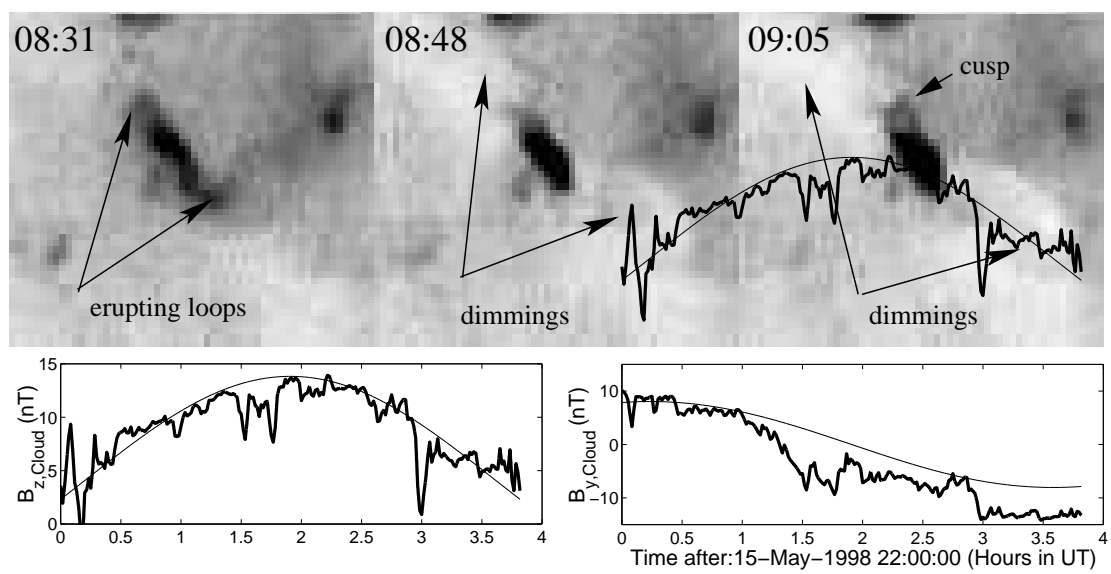

Fig. 10. Example of an eruptive event observed in the corona and in the interplanetary space. Top panels. Evolution of the coronal loops during an eruptive event in a tiny magnetic bipole (EIT, $195 \AA$ ). The contrast is reversed so reduced emission, such as dimmings, are in white. This eruption is comparable to some large-scale eruptive events, with a spatial scaling factor. Bottom panels. Magnetic field components of the associated MC in the local MC coordinates $\left(B_{z}\right.$ : axial, $B_{y}$ : orthogonal to both the spacecraft trajectory and the flux rope axis). Data are drawn with thick lines and the fitted model (Lundquist's model) with thin lines (from Mandrini et al., 2005).

coronal helicities are in better agreement when individual events are studied, as expected (despite fully different measurements and models are involved!). Still much work is needed in both domains to decrease the large uncertainties remaining, and to remove the systematic errors (leading to an expected, for different reasons, underestimation of helicities in both domains).

\section{Perspectives}

Great progress has been achieved from theoretical studies towards computing magnetic helicity from observations as summarized in this paper. Before $\approx 2000$, so just six years ago, I would have said that magnetic helicity is only an important theoretical concept to develop models! Now several methods have been developed and applied to a large set of data to compute the photospheric rate (or flux) of magnetic helicity. We are now in the position to have detailed maps of the helicity flux density. At the coronal level, magnetic extrapolation with linear force-free fields have allowed to estimate the order of magnitude, while the best is still to come with non-linear extrapolations and also from coronal loop mapping. In the interplanetary space, methods have also been developed to compute magnetic helicity in a subset of ICMEs, magnetic clouds (MCs).

The conservative property of magnetic helicity is a very important property 
to relate the measurements in various regions: photosphere, corona and interplanetary space. In particular it allows us to link very different kind of measurements, remote sensing but extended for the photosphere, in-situ but local in the interplanetary space. Moreover, the computation of the coronal helicity ejected in a CME involves a modeling that is different from the one required to compute the helicity in the corresponding magnetic cloud. Then comparing the amount of magnetic helicity involved in the corona and in the corresponding interplanetary event is a test of both the observing and modelling methodologies. This cross-check is an important step which should be extended to a larger set of events while improving the precision in both domains. This will link more quantitatively the two domains, with an expected feed back in both, for example on the structure of the magnetic configuration involved in a CME (eruption of a sheared arcade or of a twisted flux tube?).

Magnetic helicity could also play a major physical role in flares and CMEs. No consensus presently exits on its role. Then computing the evolution of magnetic helicity in various observed cases will put constraints on the models. For example, in a given AR has the photospheric flux density of helicity a given sign? Is there a larger amount of magnetic energy released when a flux tube of opposite magnetic helicity emerges and reconnects in an AR?

The work done, for deriving magnetic helicity from observations, has stimulated the development of more accurate methods to derive the photospheric velocities. These advances can be directly used to measure other quantities, such as the magnetic energy flux. However, this flux still remains more difficult to measure accurately than the magnetic helicity flux since it involves the transverse field $\mathbf{B}_{t}$ rather than $\mathbf{A}_{p}$, which can be derived from the more accurately measured normal component, $B_{n}$, when close to the solar disk centre!

The next steps involve a further theoretical exploration of the multi-faceted aspect of magnetic helicity to better incorporate all possible sources of observations. The next decade is promising both because of the higher temporal and spatial resolutions available, and because of the large range of wavelengths covered (Hinode, Solar Dynamic Observatory, as well as ground based observatories). In particular, magnetograms with high spatial resolution, resolving a larger fraction of the magnetic flux tubes will improve further the helicity rate measurements. White light observations, with their ability to detect small-scale structures even in sunspots, will also be an important complement to derive footpoint motions. Vector magnetograms with an unprecedent temporal cadence, together with a new generation of extrapolation techniques, will allow to compute the coronal field and its magnetic helicity. The detection of coronal loops in a broad range of temperatures will be another way to access the coronal helicity. Moreover, the STEREO mission will have stereoscopic images of the corona, so it will permit the removal of the ambiguity in the loop crossing (with optically thin loops, a single view does not per- 
mit a determination of which loop is in front). Finally, this mission will also provide multispacecraft observations of some MCs, giving the first insight of the field structure along the flux rope. All together with MHD simulations of the processes involved, this will hopefully clarify the precise role of magnetic helicity.

Acknowledgments: I thank Mitchell Berger, Cristina Mandrini, Peter Schuck, Mei Zhang, and the two referees for their help in improving the manuscript.

\section{References}

Abbett, W. P., Fisher, G. H., Fan, Y., 2000. The Three-dimensional Evolution of Rising, Twisted Magnetic Flux Tubes in a Gravitationally Stratified Model Convection Zone. ApJ, 540, 548-562.

Abramenko, V. I., Wang, T., Yurchishin, V. B., 1996. Analysis of Electric Current Helicity in Active Regions on the Basis of Vector Magnetograms. Solar Physics, 168, 75-89.

Alexakis, A., Mininni, P. D., Pouquet, A., 2006. On the Inverse Cascade of Magnetic Helicity. ApJ, 640, 335-343.

Amari, T., Luciani, J. F., Aly, J. J., Mikic, Z., Linker, J., 2003a. Coronal Mass Ejection: Initiation, Magnetic Helicity, and Flux Ropes. I. Boundary Motion-driven Evolution. ApJ, 585, 1073-1086.

Amari, T., Luciani, J. F., Aly, J. J., Mikic, Z., Linker, J., 2003b. Coronal Mass Ejection: Initiation, Magnetic Helicity, and Flux Ropes. II. Turbulent Diffusion-driven Evolution. ApJ, 595, 1231-1250.

Bao, S., Zhang, H., 1998. Patterns of Current Helicity for the Twenty-second Solar Cycle. ApJ, 496, L43-L46.

Bao, S. D., Pevtsov, A. A., Wang, T. J., Zhang, H. Q., 2000. Helicity Computation Using Observations From two Different Polarimetric Instruments. Solar Physics, 195, 75-87.

Barnes, D. C., 1988. Mechanical injection of magnetic helicity. Physics of Fluids 31, 2214-2220.

Berger, M. A., 1984. Rigorous new limits on magnetic helicity dissipation in the solar corona. Geophys. Astrophys. Fluid. Dyn. 30, 79-104.

Berger, M. A., 1985. Structure and stability of constant-alpha force-free fields. ApJS, 59, 433-444.

Berger, M. A., 1988. An energy formula for nonlinear force-free magnetic fields. A\&A, 201, 355-361.

Berger, M. A., 2003. Topological quantities in magnetohydrodynamics. In: Advances in Nonlinear Dynamics. pp. 345-383.

Berger, M. A., Field, G. B., 1984. The topological properties of magnetic helicity. J. Fluid. Mech. 147, 133-148. 
Berger, T. E., Lites, B. W., 2003. Weak-Field Magnetogram Calibration using Advanced Stokes Polarimeter Flux Density Maps - II. SOHO/MDI Full-Disk Mode Calibration. Solar Physics, 213, 213-229.

Bothmer, V., Schwenn, R., 1994. Eruptive prominences as sources of magnetic clouds in the solar wind. Space Science Reviews 70, 215-220.

Brandenburg, A., 2001. The Inverse Cascade and Nonlinear Alpha-Effect in Simulations of Isotropic Helical Hydromagnetic Turbulence. ApJ, 550, 824840.

Brandenburg, A., Subramanian, K., 2005. Astrophysical magnetic fields and nonlinear dynamo theory. Phys. Rep. 417, 1-209.

Brown, M., Canfield, R., Pevtsov, A., 1999. Magnetic Helicity in Space and Laboratory Plasmas. Geophy. Mon. Ser. 111, AGU.

Burlaga, L. F., 1995. Interplanetary magnetohydrodynamics. New York : Oxford University Press, 1995.

Chae, J., 2001. Observational Determination of the Rate of Magnetic Helicity Transport through the Solar Surface via the Horizontal Motion of Field Line Footpoints. ApJ, 560, L95-L98.

Chae, J., Moon, Y.-J., Park, Y.-D., 2004. Determination of magnetic helicity content of solar active regions from SOHO/MDI magnetograms. Solar Physics, 223, 39-55.

Chae, J., Moon, Y.-J., Rust, D. M., Wang, H., Goode, P. R., 2003. Magnetic Helicity Pumping by Twisted Flux Tube Expansion. Journal of Korean Astronomical Society 36, 33-41.

Chae, J., Wang, H., Qiu, J., Goode, P. R., Strous, L., Yun, H. S., 2001. The Formation of a Prominence in Active Region NOAA 8668. I. SOHO/MDI Observations of Magnetic Field Evolution. ApJ, 560, 476-489.

Cheung, M., Schüssler, M., Moreno-Insertis, F., 2005. 3D Magneto-Convection and Flux Emergence in the Photosphere. In: Innes, D. E., Lagg, A., Solanki, S. A. (Eds.), ESA SP-596: Chromospheric and Coronal Magnetic Fields. p. 54.1 .

Cid, C., Hidalgo, M. A., Nieves-Chinchilla, T., Sequeiros, J., Viñas, A. F., 2002. Plasma and Magnetic Field Inside Magnetic Clouds: a Global Study. Solar Physics, 207, 187-198.

Dasso, S., Mandrini, C. H., Démoulin, P., Farrugia, C. J., 2003. Magnetic helicity analysis of an interplanetary twisted flux tube. JGR, 108, SSH 3-1.

Dasso, S., Mandrini, C. H., Démoulin, P., Luoni, M. L., 2006. A new modelindependent method to compute magnetic helicity in magnetic clouds. A\&A, 455, 349-359.

Dasso, S., Mandrini, C. H., Démoulin, P., Luoni, M. L., Gulisano, A. M., 2005. Large scale MHD properties of interplanetary magnetic clouds. Advances in Space Research 35, 711-724.

Démoulin, P., Berger, M. A., 2003. Magnetic Energy and Helicity Fluxes at the Photospheric Level. Solar Physics, 215, 203-215.

Démoulin, P., Mandrini, C. H., van Driel-Gesztelyi, L., et al., 2002a. What is the source of the magnetic helicity shed by CMEs? The long-term helicity 
budget of AR 7978. A\&A, 382, 650-665.

Démoulin, P., Mandrini, C. H., Van Driel-Gesztelyi, L., Lopez Fuentes, M. C., Aulanier, G., 2002b. The Magnetic Helicity Injected by Shearing Motions. Solar Physics, 207, 87-110.

Démoulin, P., Pariat, E., Berger, M. A., 2006. Basic Properties of Mutual Magnetic Helicity. Solar Physics, 233, 3-27.

DeVore, C. R., 2000. Magnetic Helicity Generation by Solar Differential Rotation. ApJ, 539, 944-953.

Elsasser, W. M., 1956. Hydromagnetic Dynamo Theory. Reviews of Modern Physics 28, 135-163.

Emonet, T., Moreno-Insertis, F., 1998. The Physics of Twisted Magnetic Tubes Rising in a Stratified Medium: Two-dimensional Results. ApJ, 492, 804-821.

Farrugia, C. J., Janoo, L. A., Torbert, R. B., et al., 1999. A Uniform-Twist Magnetic Flux Rope in the Solar Wind. In: AIP Conf. Proc. 471: Solar Wind Nine. pp. 745-748.

Finn, J. H., Antonsen, T. M. J., 1985. Magnetic helicity: What is it, and what is it good for? Comments Plasma Phys. Contr. Fusion 9, 111-126.

Freedman, M. H., Berger, M. A., 1993. Combinatorial relaxation of magnetic fields. Geophys. Astrophys. Fluid. Dyn. 73, 91-96.

Frisch, U., Pouquet, A., Leorat, J., Mazure, A., 1975. Possibility of an inverse cascade of magnetic helicity in magnetohydrodynamic turbulence. Journal of Fluid Mechanics 68, 769-778.

Georgoulis, M. K., LaBonte, B. J., 2006. Reconstruction of an Inductive Velocity Field Vector from Doppler Motions and a Pair of Solar Vector Magnetograms. ApJ, 636, 475-495.

Gibson, S. E., Fan, Y., Mandrini, C., Fisher, G., Démoulin, P., 2004. Observational Consequences of a Magnetic Flux Rope Emerging into the Corona. ApJ, 617, 600-613.

Gosling, J. T., Baker, D. N., Bame, S. J., Feldman, W. C., Zwickl, R. D., Smith, E. J., 1987. Bidirectional solar wind electron heat flux events. JGR, 92, 8519-8535.

Green, L. M., López fuentes, M. C., Mandrini, C. H., et al., 2002. The Magnetic Helicity Budget of a CME-Prolific Active Region. Solar Physics, 208, 43-68.

Gulisano, A. M., Dasso, S., Mandrini, C. H., Démoulin, P., 2005. Magnetic clouds: A statistical study of magnetic helicity. J. of Atmosph. and Solar Terrestr. Phys. 67, 1761-1766.

Hagino, M., Sakurai, T., 2004. Latitude Variation of Helicity in Solar Active Regions. PASJ, 56, 831-843.

Hartkorn, K., Wang, H., 2004. Magnetic Helicity Change Rate Associated with X-Class and M-Class Flares. Solar Physics, 225, 311-324.

Heyvaerts, J., Priest, E. R., 1984. Coronal heating by reconnection in DC current systems - A theory based on Taylor's hypothesis. A\&A, 137, 63-78.

Hidalgo, M. A., Cid, C., Vinas, A. F., Sequeiros, J., 2002. A non-force-free approach to the topology of magnetic clouds in the solar wind. JGR, 107, 
SSH 1-1.

Hornig, G., 2006. A Universal Magnetic Helicity Integral. ArXiv Astrophysics e-prints, astro-ph/0606694.

Hu, Q., Sonnerup, B. U. Ö., 2002. Reconstruction of magnetic clouds in the solar wind: Orientations and configurations. JGR, 107, SSH 10-1.

Jacobs, C., Poedts, S., van der Holst, B., 2006. The effect of the solar wind on CME triggering by magnetic foot point shearing. A\&A, 450, 793-803.

Kusano, K., Maeshiro, T., Yokoyama, T., Sakurai, T., 2002. Measurement of Magnetic Helicity Injection and Free Energy Loading into the Solar Corona. ApJ, 577, 501-512.

Kusano, K., Maeshiro, T., Yokoyama, T., Sakurai, T., 2004a. Study of Magnetic Helicity in the Solar Corona. In: Sakurai, T., Sekii, T. (Eds.), ASP Conf. Ser. 325: The Solar-B Mission and the Forefront of Solar Physics. pp. 175-184.

Kusano, K., Maeshiro, T., Yokoyama, T., Sakurai, T., 2004b. The Trigger Mechanism of Solar Flares in a Coronal Arcade with Reversed Magnetic Shear. ApJ, 610, 537-549.

Larson, D. E., Lin, R. P., McTiernan, J. M., et al., 1997. Tracing the topology of the October 18-20, 1995, magnetic cloud with $0.1-10^{2} \mathrm{keV}$ electrons. GRL, 24, 1911-1914.

Lepping, R. P., Burlaga, L. F., Jones, J. A., 1990. Magnetic field structure of interplanetary magnetic clouds at 1 AU. JGR, 95, 11957-11965.

Longcope, D. W., 2004. Inferring a Photospheric Velocity Field from a Sequence of Vector Magnetograms: The Minimum Energy Fit. ApJ, 612, 11811192.

Longcope, D. W., Welsch, B. T., 2000. A Model for the Emergence of a Twisted Magnetic Flux Tube. ApJ, 545, 1089-1100.

Low, B. C., 1997. The role of coronal mass ejections in solar activity. In: Coronal Mass Ejection, Geophys. Monograph 99. pp. 39-48.

Low, B. C., 2006. Magnetic Helicity in a Two-Flux Partitioning of an Ideal Hydromagnetic Fluid. ApJ, 646, 1288-1302.

Luoni, M. L., Mandrini, C. H., Dasso, S., van Driel-Gesztelyi, L., Démoulin, P., 2005. Tracing magnetic helicity from the solar corona to the interplanetary space. Journal of Atmospheric and Terrestrial Physics 67, 1734-1743.

Lynch, B. J., Gruesbeck, J. R., Zurbuchen, T. H., Antiochos, S. K., 2005. Solar cycle-dependent helicity transport by magnetic clouds. JGR, 110, 81078117.

Magara, T., Longcope, D. W., 2003. Injection of Magnetic Energy and Magnetic Helicity into the Solar Atmosphere by an Emerging Magnetic Flux Tube. ApJ, 586, 630-649.

Mandrini, C. H., Pohjolainen, S., Dasso, S., et al., 2005. Interplanetary flux rope ejected from an X-ray bright point. The smallest magnetic cloud source-region ever observed. A\&A, 434, 725-740.

Marubashi, K., 1997. Interplanetary Magnetic Flux Ropes and Solar Filaments. In: Coronal Mass Ejection, Geophys. Monograph 99. pp. 147-156. 
Matthaeus, W. H., Goldstein, M. L., 1982. Measurement of the rugged invariants of magnetohydrodynamic turbulence in the solar wind. JGR, 87, 6011-6028.

Matthaeus, W. H., Goldstein, M. L., Smith, C., 1982. Evaluation of magnetic helicity in homogeneous turbulence. Physical Review Letters 48, 1256-1259.

Metcalf, T. R., Jiao, L., McClymont, A. N., Canfield, R. C., Uitenbroek, H., 1995. Is the solar chromospheric magnetic field force-free? ApJ, 439, 474481.

Moffatt, H., 1969. The degree of knottedness of tangled vortex lines. J. Fluid Mech. 35, 117-129.

Moon, Y.-J., Chae, J., Choe, G. S., et al., 2002a. Flare Activity and Magnetic Helicity Injection by Photospheric Horizontal Motions. ApJ, 574, 10661073.

Moon, Y.-J., Chae, J., Wang, H., Choe, G. S., Park, Y. D., 2002b. Impulsive Variations of the Magnetic Helicity Change Rate Associated with Eruptive Flares. ApJ, 580, 528-537.

Nindos, A., Andrews, M. D., 2004. The Association of Big Flares and Coronal Mass Ejections: What Is the Role of Magnetic Helicity? ApJ, 616, L175L178.

Nindos, A., Zhang, H., 2002. Photospheric Motions and Coronal Mass Ejection Productivity. ApJ, 573, L133-L136.

Nindos, A., Zhang, J., Zhang, H., 2003. The Magnetic Helicity Budget of Solar Active Regions and Coronal Mass Ejections. ApJ, 594, 1033-1048.

November, L. J., Simon, G. W., 1988. Precise proper-motion measurement of solar granulation. ApJ, 333, 427-442.

Pariat, E., Démoulin, P., Berger, M. A., 2005. Photospheric flux density of magnetic helicity. A\&A, 439, 1191-1203.

Pariat, E., Nindos, A., Démoulin, P., Berger, M. A., 2006. What is the spatial distribution of magnetic helicity injected in a solar active region? A\&A, 452, 623-630.

Pevtsov, A. A., 2000. Transequatorial Loops in the Solar Corona. ApJ, 531, $553-560$.

Pevtsov, A. A., Canfield, R. C., Metcalf, T. R., 1995. Latitudinal variation of helicity of photospheric magnetic fields. ApJ, 440, L109-L112.

Pevtsov, A. A., Maleev, V. M., Longcope, D. W., 2003. Helicity Evolution in Emerging Active Regions. ApJ, 593, 1217-1225.

Phillips, A. D., MacNeice, P. J., Antiochos, S. K., 2005. The Role of Magnetic Helicity in Coronal Mass Ejections. ApJ, 624, L129-L132.

Potts, H. E., Barrett, R. K., Diver, D. A., 2004. Balltracking: An highly efficient method for tracking flow fields. A\&A, 424, 253-262.

Qiu, J., Gary, D. E., 2003. Flare-related Magnetic Anomaly with a Sign Reversal. ApJ, 599, 615-625.

Régnier, S., Amari, T., Canfield, R. C., 2005. Self and mutual magnetic helicities in coronal magnetic configurations. A\&A, 442, 345-349.

Riley, P., Linker, J. A., Mikić, Z., et al., 2003. Using an MHD simulation 
to interpret the global context of a coronal mass ejection observed by two spacecraft. JGR, 108, 2-1.

Romano, P., Contarino, L., Zuccarello, F., 2005. Observational evidence of the primary role played by photospheric motions in magnetic helicity transport before a filament eruption. A\&A, 433, 683-690.

Russell, C. T., Shinde, A. A., 2005. On Defining Interplanetary Coronal Mass EJECTIONs from Fluid Parameters. Solar Physics, 229, 323-344.

Rust, D. M., 1994. Spawning and shedding helical magnetic fields in the solar atmosphere. GRL, 21, 241-244.

Schuck, P. W., 2005. Local Correlation Tracking and the Magnetic Induction Equation. ApJ, 632, L53-L56.

Schuck, P. W., 2006. Tracking Magnetic Footpoints with the Magnetic Induction Equation. ApJ, 646, 1358-1391.

Strous, L. H., Scharmer, G., Tarbell, T. D., Title, A. M., Zwaan, C., 1996. Phenomena in an emerging active region. I. Horizontal dynamics. A\&A, 306, 947-959.

Taylor, J. B., 1974. Relaxation of Toroidal Plasma and Generation of Reverse Magnetic Fields. Physical Review Letters 33, 1139-1141.

van-Driel Gesztelyi, L., Démoulin, P., Mandrini, C. H., 2003. Observations of Magnetic Helicity. Advances in Space Research 32, 1855-1866.

Vandas, M., Romashets, E. P., 2003. A force-free field with constant alpha in an oblate cylinder: A generalization of the Lundquist solution. A\&A, 398, 801-807.

Wang, J., 1996. A Note on the Evolution of Magnetic Helicity in Active Regions. Solar Physics, 163, 319-325.

Welsch, B. T., Fisher, G. H., Abbett, W. P., Regnier, S., 2004. ILCT: Recovering Photospheric Velocities from Magnetograms by Combining the Induction Equation with Local Correlation Tracking. ApJ, 610, 1148-1156.

Welsch, B. T., Longcope, D. W., 2003. Magnetic Helicity Injection by Horizontal Flows in the Quiet Sun. I. Mutual-Helicity Flux. ApJ, 588, 620-629.

Yamamoto, T. T., Kusano, K., Maeshiro, T., Yokoyama , T., Sakurai, T., 2005. Magnetic Helicity Injection and Sigmoidal Coronal Loops. ApJ, 624, 1072-1079.

Yurchyshyn, V. B., Wang, H., Goode, P. R., Deng, Y., 2001. Orientation of the Magnetic Fields in Interplanetary Flux Ropes and Solar Filaments. ApJ, 563, 381-388.

Zhang, M., Flyer, N., Low, B. C., 2006. Magnetic Field Confinement in the Corona: The Role of Magnetic Helicity Accumulation. ApJ, 644, 575-586.

Zhang, M., Low, B. C., 2003. Magnetic Flux Emergence into the Solar Corona. III. The Role of Magnetic Helicity Conservation. ApJ, 584, 479-496.

Zhao, X. P., Hoeksema, J. T., Marubashi, K., 2001. Magnetic cloud Bs events and their dependence on cloud parameters. JGR, 106, 15643-15656. 